\title{
Compositional Restricted Boltzmann Machines Unveil the Brain-Wide Organization of Neural Assemblies
}

\author{
Thijs L. van der Plas ${ }^{\mathrm{a}, \mathrm{b}, \mathrm{c}, *}$, Jérôme Tubiana ${ }^{\mathrm{d}, *}$, Guillaume Le Goc ${ }^{\mathrm{b}}$, Geoffrey Migault ${ }^{\mathrm{b}}$, Michael Kunst ${ }^{\mathrm{e}, \mathrm{f}}$, \\ Herwig Baier ${ }^{\mathrm{e}}$, Volker Bormuth ${ }^{\mathrm{b}, \mathrm{g}, * *}$, Bernhard Englitz ${ }^{\mathrm{a}, \mathrm{g}, * *}$, Georges Debrégeas ${ }^{\mathrm{b}, \mathrm{g}, * *}$ \\ ${ }^{a}$ Computational Neuroscience Lab, Department of Neurophysiology, Donders Center for Neuroscience, Radboud University, Nijmegen, \\ The Netherlands \\ ${ }^{b}$ Sorbonne Université, CNRS, Institut de Biologie Paris-Seine (IBPS), Laboratoire Jean Perrin (LJP), Paris, France \\ ${ }^{c}$ Department of Physiology, Anatomy and Genetics, University of Oxford, Oxford, United Kingdom \\ ${ }^{d}$ Blavatnik School of Computer Science, Tel Aviv University, Tel Aviv \\ ${ }^{e}$ Department Genes - Circuits - Behavior, Max Planck Institute of Neurobiology, 82152 Martinsried, Germany \\ ${ }^{f}$ Allen Institute for Brain Science, Seattle, WA 98109, USA \\ ${ }^{g}$ Corresponding author
}

\begin{abstract}
Patterns of endogenous activity in the brain reflect a stochastic exploration of the neuronal state space that is constrained by the underlying assembly organization of neurons. Yet it remains to be shown that this interplay between neurons and their assembly dynamics indeed suffices to generate whole-brain data statistics. Here we recorded the activity from $\sim 40,000$ neurons simultaneously in zebrafish larvae, and show that a data-driven network model of neuron-assembly interactions can accurately reproduce the mean activity and pairwise correlation statistics of their spontaneous activity. This model, the compositional Restricted Boltzmann Machine, unveils $\sim 200$ neural assemblies, which compose neurophysiological circuits and whose various combinations form successive brain states. From this, we mathematically derived an interregional functional connectivity matrix, which is conserved across individual animals and correlates well with structural connectivity. This novel, assembly-based generative model of brain-wide neural dynamics enables physiology-bound perturbation experiments in silico.
\end{abstract}

Keywords: Light-Sheet Microscopy, Zebrafish, Neural Assembly, Maximum Entropy, Restricted Boltzmann Machine, Functional Connectivity

\section{Introduction}

The brain is a highly connected network, organized across multiple scales, from local circuits involving just a few neurons to extended networks spanning multiple brain regions (White et al., 1986, Song et al., 2005; Kunst et al. 2019). Concurrent with this spatial organization, brain activity exhibits correlated firing among

${ }_{5}$ large groups of neurons, often referred to as neural assemblies (Harris 2005). This assembly organization of brain dynamics has been observed in, e.g., auditory cortex (Bathellier et al. 2012), motor cortex (Narayanan et al. 2005), prefrontal cortex (Tavoni et al., 2017), hippocampus (Lin et al. 2005), retina (Shlens et al., 2009), and zebrafish optic tectum (Romano et al. 2015; Mölter et al. 2018; Diana et al., 2019; Triplett et al. 2020). These neural assemblies are thought to form elementary computational units and subserve essential cognitive functions such as short-term memory, sensorimotor computation or decision-making (Hebb, 1949, Gerstein et al., 1989, Harris, 2005; Buzsáki, 2010, Harris, 2012, Palm et al., 2014, Eichenbaum, 2018). Despite the

\footnotetext{
${ }^{*}$ These authors contributed equally.

** Senior authors with equal contribution

Email addresses: volker . bormuth@upmc.fr (Volker Bormuth), b.englitz@donders.ru.nl (Bernhard Englitz), georges.debregeas@upmc.fr (Georges Debrégeas)
} 
prevalence of these assemblies across the nervous system and their role in neural computation, it remains an open challenge to extract the assembly organization of a full brain and to show that the assembly activity state, derived from that of the neurons, is sufficient to account for the collective neural dynamics.

The need to address this challenge is catalyzed by technological advances in light-sheet microscopy, enabling the simultaneous recording of the majority of neurons in the zebrafish brain at single-cell resolution in vivo (Panier et al., 2013; Ahrens et al., 2013; Wolf et al. 2015, 2017; Migault et al., 2018; Vanwalleghem et al. 2018). This neural recording technique opens up new avenues for constructing near-complete models of neural activity, and in particular its assembly organization. Recent attempts have been made to identify assemblies using either clustering (Panier et al. 2013; Triplett et al. 2018, Chen et al., 2018; Mölter et al. 2018), dimensionality reduction approaches (Romano et al., 2015; Mu et al. 2019) or latent variable models (Diana et al., 2019, Triplett et al. 2020), albeit often limited to single brain regions. However, these methods do not explicitly assess to what extent the inferred assemblies could give rise to the observed neural data statistics, which is a crucial property of physiologically meaningful assemblies (Harris. 2005). Here, we address this challenge by developing a generative model of neural activity that is explicitly constrained by the assembly organization, thereby quantifying if assemblies indeed suffice to produce the observed neural data statistics.

Specifically, we formalize neural assemblies using a bipartite network of two connected layers representing the neuronal and the assembly activity, respectively. Together with the maximum entropy principle (Jaynes. 1957, Bialek, 2012), this architecture defines the Restrictive Boltzmann Machine (RBM) model (Hinton \& Salakhutdinov, 2006). Here we use an extension to the classical RBM definition termed compositional RBM (cRBM) that we have recently introduced (Tubiana \& Monasson, 2017; Tubiana et al., 2019a) and which brings multiple advances to assembly-based network modeling: (1) The maximum entropy principle ensures that neural assemblies are inferred solely from the data statistics, in contrast to other approaches where ad hoc constraints are imposed. (2) The generative nature of the model, through alternate data sampling of the neuronal and assembly layers, can be leveraged to evaluate its capacity to replicate the empirical data statistics. (3) The cRBM steers the assembly organization to the so-called compositional phase where a small number of assemblies are active at any point in time, making the resulting model highly interpretable as we have shown previously for protein sequence analysis (Tubiana et al. 2019b).

Here, we have successfully trained cRBMs to brain-scale, neuron-level recordings of spontaneous activity in larval zebrafish containing 41000 neurons on average (Panier et al., 2013; Wolf et al., 2017, Migault et al., 2018). This represents an increase of $\sim 2$ orders of magnitude in number of neurons with respect to previously 45 reported RBM implementations (Köster et al., 2014; Gardella et al., 2017; Volpi et al., 2020), attained through significant algorithmic and computational enhancements. We found that all cells could be grouped into 100-200 partially overlapping assemblies, which are anatomically localized and together span the entire brain, and accurately replicate the first and second order statistics of the neural activity. These assemblies were found to carry more predictive power than a fully connected model which has orders of magnitude more parameters, validating that assemblies underpin collective neural dynamics. Further, the probabilistic nature of our model allowed us to compute a functional connectivity matrix by conditioning the probability of a neuron to fire on the network state. This assembly-based functional connectivity is well-conserved across individual fish and consistent with anatomical connectivity at the mesoscale (Kunst et al. 2019).

In summary, we present an assembly decomposition spanning the zebrafish brain, which accurately accounts for its activity statistics. Our cRBM model provides a widely applicable tool to the community to construct low-dimensional data representations that are defined by the statistics of the data, in particular for very high-dimensional systems. A future application of such models is to predict the whole-brain response to the modulation of the activity of particular assemblies, thus creating hypotheses for future experiments. 


\section{Results}

\subsection{Compositional RBMs construct Hidden Units by grouping neurons into assemblies}

Spontaneous neural activity was recorded from 8 zebrafish larvae aged 5-7 days post fertilization expressing the GCaMP6s or GCaMP6f calcium reporters using light-sheet microscopy (Panier et al., 2013; Wolf et al., 2017, Migault et al., 2018). Each data set contained the activity of a large fraction of the neurons in the brain (40709 \pm 13854; mean \pm standard deviation), which, after cell segmentation, were registered onto the ZBrain atlas (Randlett et al., 2015) and mapzebrain atlas (Kunst et al., 2019). Individual neuronal fluorescence traces were deconvolved to binarized spike trains using blind sparse deconvolution (Tubiana et al., 2020). This data acquisition process is depicted in Figure 1 A.

We trained compositional Restricted Boltzmann Machine (cRBM) models to capture the activity statistics of these neural recordings. cRBMs are maximum entropy models, i.e. the maximally unconstrained solution that fits model-specific data statistics (Hinton \& Salakhutdinov, 2006, Tubiana \& Monasson, 2017, Gardella et al. 2019), and critically extend the classical RBM formulation. Its architecture consists of a bipartite graph where the high-dimensional layer of neurons $\mathbf{v}$ (named 'visible units' in RBM terminology) is connected to the low-dimensional layer of latent components, termed Hidden Units (HUs) $\mathbf{h}$. Their interaction is characterized by a weight matrix $\mathbf{W}$ that is regularized to be sparse. The collection of neurons that have non-zero interactions with a particular HU, noted $h_{\mu}$ (i.e. with $\left|w_{i, \mu}\right|>0$ ), define its corresponding neural assembly $\mu$ (Figure $\left.1 \mathrm{~B}\right)$. This weight matrix, together with the neuron weight vector $\mathbf{g}$ and $\mathrm{HU}$ potential $\mathcal{U}$, defines the transformation from the binarized neural activity $\mathbf{v}(t)$ to the continuous HU activity $\mathbf{h}(t)$ (Figure $1 \mathrm{~B}$ ). Figure $1 \mathrm{C}$ shows all recorded neurons of a zebrafish brain, color-labeled according to their strongest-connecting HU, illustrating that cRBMinferred assemblies are typically densely localized in space and together span the entire brain.

Beyond its architecture (Figure 2A), the model is defined by the probability function $P(\mathbf{v}, \mathbf{h})$ of any data configuration $(\mathbf{v}, \mathbf{h})$ (see STAR Methods for details):

$$
P(\mathbf{v}, \mathbf{h})=\frac{1}{Z} \exp (-E(\mathbf{v}, \mathbf{h}))
$$

Where $Z$ is the partition function that normalizes Equation 1 and $E$ is the following energy function:

$$
E(\mathbf{v}, \mathbf{h})=-\sum_{i} g_{i} v_{i}+\sum_{\mu} \mathcal{U}_{\mu}\left(h_{\mu}\right)-\sum_{i, \mu} w_{i, \mu} v_{i} h_{\mu}
$$

HU activity $\mathbf{h}$ is obtained by sampling from the conditional probability function $P(\mathbf{h} \mid \mathbf{v})$ :

$$
P(\mathbf{h} \mid \mathbf{v})=\prod_{\mu=1}^{M} P\left(h_{\mu} \mid \mathbf{v}\right) \propto \prod_{\mu=1}^{M} \exp \left(-\mathcal{U}_{\mu}\left(h_{\mu}\right)+h_{\mu} \cdot \sum_{i} w_{i, \mu} v_{i}\right)
$$

Conversely, neural activity is obtained from HU activity through:

$$
P(\mathbf{v} \mid \mathbf{h})=\prod_{i=1}^{N} P\left(v_{i} \mid \mathbf{h}\right) \propto \prod_{i=1}^{N} \exp \left(g_{i} v_{i}+v_{i} \cdot \sum_{\mu} w_{i, \mu} h_{\mu}\right)
$$

Equations 3 and 4 mathematically reflect the dual relationship between neural and assembly states: the Hidden Units $\mathbf{h}$ drive 'visible' neural activity $\mathbf{v}$, expressed as $P(\mathbf{v} \mid \mathbf{h})$, while the stochastic assembly activity $\mathbf{h}$ itself is defined as a function of the activity of the neurons: $P(\mathbf{h} \mid \mathbf{v})$. Importantly, the model does not include direct connections between neurons, hence neural correlations $\left\langle v_{i} v_{j}\right\rangle$ can arise solely from shared assemblies. Moreover, this bipartite architecture ensures that the conditional distributions factorize, leading to a sampling procedure where all neurons or all HUs can be sampled in parallel. The cRBM leverages this property to efficiently generate new data by Monte Carlo sampling alternately from $P(\mathbf{h} \mid \mathbf{v})$ and $P(\mathbf{v} \mid \mathbf{h})$ (Figure $2 \mathrm{~B}$ ). 
The cRBM differs from the classical RBM formulation (Hinton \& Salakhutdinov, 2006) through the introduction of double Rectified Linear Unit ( $\mathrm{dReLU}$ ) potentials $\overline{\mathcal{U}}_{\mu}$, weight sparsity regularization and normalized HU activity (further detailed in STAR Methods). We have previously demonstrated in theory and application (Tubiana \& Monasson, 2017, Tubiana et al., 2019a|b) that this new formulation steers the model into the socalled compositional phase, which makes the latent representation highly interpretable. This phase occurs when a limited number $m$ of HUs co-activate such that $1 \ll m \ll M$ where $M$ is the total number of HUs. Thus, each visible configuration is mapped to a specific combination of activated HUs. This contrasts with the ferromagnetic phase $(m \sim 1)$ where each HU encodes one specific activity pattern, thus severely limiting the possible number of encoded patterns, or the spin-glass phase $(m \sim M)$ where all HUs activate simultaneously, yielding a very complex assembly patchwork (Tubiana \& Monasson, 2017). Therefore, the compositional phase can provide the right level of granularity for a meaningful interpretation of the cRBM neural assemblies by decomposing the overall activity as a time-dependent co-activation of different assemblies of interpretable size and extent.

\subsection{Trained cRBMs accurately replicate data statistics}

cRBM models are trained to maximize the $P(\mathbf{v}, \mathbf{h}) \log$-likelihood of the zebrafish data recordings, which is achieved by matching the model-generated statistics $\left\langle v_{i}\right\rangle,\left\langle h_{\mu}\right\rangle$ and $\left\langle v_{i} h_{\mu}\right\rangle$ (the mean neuronal activity, mean HU activity and their correlations, respectively) to the empirical data statistics (Equation 15. In order to optimize the two free parameters of the cRBM model - the sparsity regularization parameter $\lambda$ and the total number of HUs $M$ - we assessed the cRBM performance for a grid of $(\lambda, M)$-values for one data set (fish \#3). This analysis yielded an optimum for $\lambda=0.02$ and $M=200$ (Fig S1). These values were subsequently used for all recordings, where $M$ was scaled with the number of neurons $N$.

We trained cRBMs on $70 \%$ of the recording length, and compared the statistics of model-generated data to the withheld test data set (the remaining 30\% of recording, see STAR Methods for details). After convergence, the cRBM generated data that replicated the training statistics accurately, with normalized Root Mean Square Error (nRMSE) values of $\mathrm{nRMSE}_{\left\langle v_{i}\right\rangle}=0.11, \mathrm{nRMSE}_{\left\langle h_{\mu}\right\rangle}=0.15$ and $\mathrm{nRMSE}_{\left\langle v_{i} h_{\mu}\right\rangle}=0.09$ (Figures $2 \mathrm{C}-\mathrm{E}$ ). Here, nRMSE is normalized such that 1 corresponds to shuffled data statistics and 0 corresponds to the best possible RMSE, i.e. between train and test data.

We further evaluated cRBM performance to assess its ability to capture data statistics that the cRBM was not explicitly trained to replicate: the pairwise correlations between neurons $\left\langle v_{i} v_{j}\right\rangle$ and the pairwise correlations between HUs $\left\langle h_{\mu} h_{\nu}\right\rangle$. We found that these statistics were also accurately replicated by model-generated data, with nRMSE $\left\langle\left\langle_{\left.v_{i} v_{j}\right\rangle}=-0.09\right.\right.$ (meaning that the model slightly outperformed the train-test data difference) and $\mathrm{nRMSE}_{\left\langle h_{\mu} h_{v}\right\rangle}=0.17$ (Figures $2 \mathrm{~F}, \mathrm{G}$ ). The fact that cRBM also accurately replicated neural correlations $\left\langle v_{i} v_{j}\right\rangle$ (Figure $2 \mathrm{~F}$ ) is of particular relevance, since this indicates that (1) the assumption that neural correlations can be explained by their shared assemblies is justified and (2) cRBMs may provide an efficient mean to model neural interactions of such large systems $\left(N \sim 10^{4}\right)$ where directly modeling all $N^{2}$ interactions would be computationally infeasible or not sufficiently constrained by the available data.

Next, we assessed the reconstruction quality after neural data was compressed by the cRBM low-dimensional bottleneck. This is important to prevent trivial, undesired solutions like $w_{i, \mu}=0 \forall i, \mu$ which would directly lead to $\left\langle h_{\mu}\right\rangle_{P(\mathbf{v}, \mathbf{h})}=\left\langle h_{\mu}\right\rangle_{\text {data }}=0$ (potentially because of strong sparsity regularization). Figure $2 \mathrm{H}$ shows the distribution of cRBM reconstruction quality of all neurons (in purple), quantified by the normalized log-likelihood (nLLH) such that 0 corresponds to an independent model $\left(P\left(v_{i}(t)\right)=\left\langle v_{i}\right\rangle\right)$ and 1 corresponds to perfect reconstruction (non-normalized LLH $=0$ ). For comparison, we also reconstructed the neural activity using a fully connected Generalized Linear Model (GLM, see STAR Methods and Figures S2, 2H, blue). The cRBM nLLH distribution is significantly greater than the GLM nLLH distribution (one-sided Mann Whitney U test, $P<10^{-42}$ ), with medians $\mathrm{LLH}_{\mathrm{cRBM}}=0.24$ and $\mathrm{LLH}_{\mathrm{GLM}}=0.20$. Hence, projecting the neural data onto the low-dimensional representation of the HUs does not compromise the ability to explain the neural activity.

In fact, reconstruction quality of the cRBM slightly outperforms the GLM, possibly due to the suppression of noise in the cRBM estimate. The optimal $(\lambda=0.02, M=200)$ choice of free parameters was selected by 


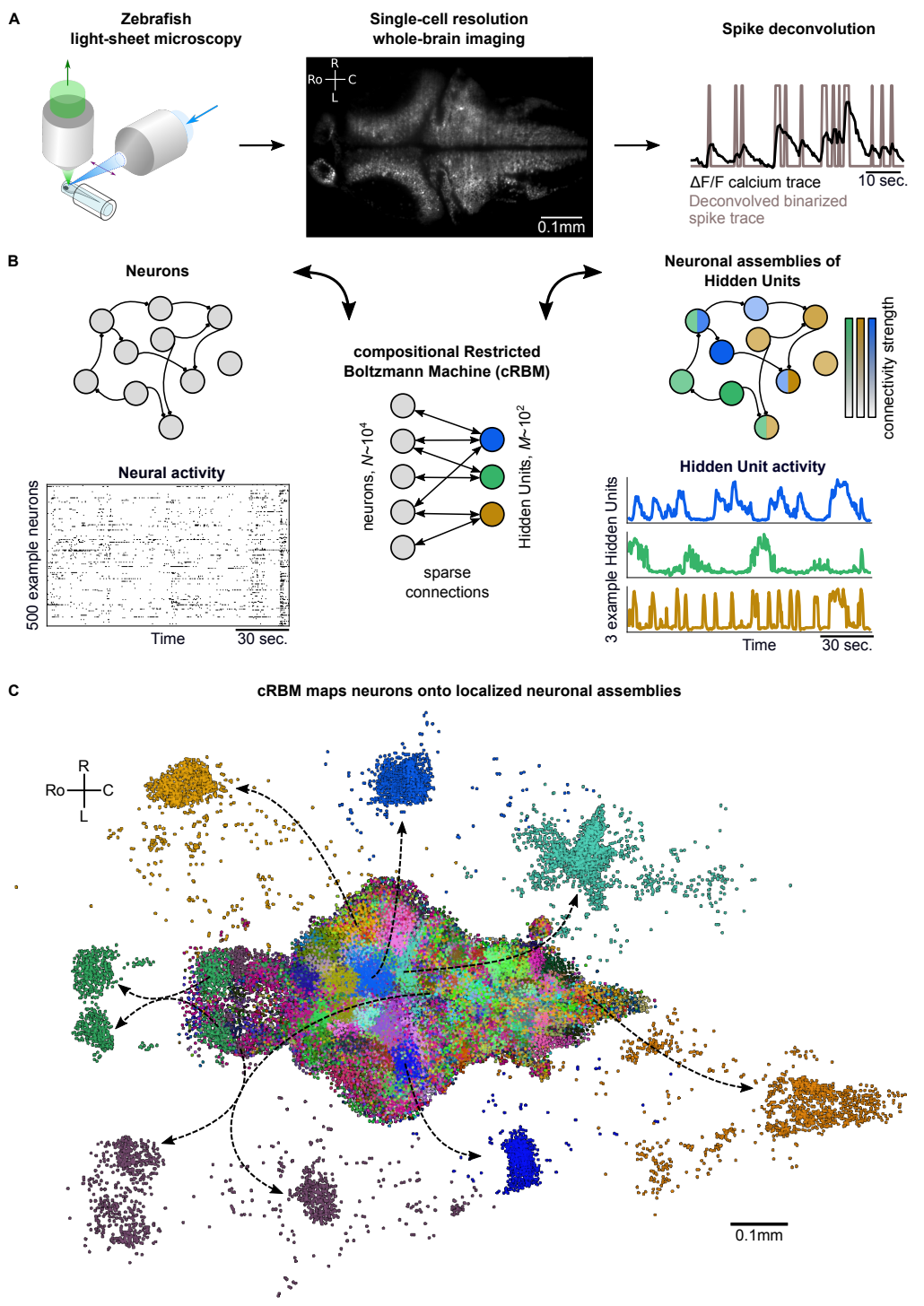

Figure 1: cRBMs construct Hidden Units by grouping neurons into assemblies

(A) The neural activity of zebrafish larvae was imaged using light-sheet microscopy (left), which resulted in brain-scale, single-cell resolution data sets (middle, microscopy image of a single plane shown for fish \#1). Calcium activity $\Delta F / F$ was deconvolved to binarized spike traces for each segmented cell (right, example neuron).

(B) cRBM sparsely connects neurons (left) to Hidden Units (HUs, right). The neurons that connect to a HU are defined to be its corresponding assembly (depicted by color labeling, right panel). Data sets typically consist of $N \sim 10^{4}$ neurons and $M \sim 10^{2}$ HUs. The activity of 500 randomly chosen example neurons (raster plot, left) and HUs 99, 26, 115 (activity traces, right) of the same time excerpt is shown. $\mathrm{HU}$ activity is continuous and is determined by transforming the neural activity of its assembly.

(C) The neural assemblies of an example data set (fish \#3) are shown by coloring each neuron according to its strongest-connecting HU. 7 assemblies are highlighted (starting rostrally at the green forebrain assembly, going clockwise: HU 177, 187, 7, 156, 124, 64, 178), by showing their neurons with a connection $|w| \geq 0.1$. See Figure 3 for more anatomical details of assemblies.

R: Right, L: Left, Ro: Rostral, C: Caudal.

cross-validating the median of the cRBM reconstruction quality, together with the normalized RMSE of the 5 previously described statistics (Figure S1).

Lastly, we confirmed that the cRBM indeed resides in the compositional phase, characterized by $1 \ll m(t) \ll$ $M$ where $m(t)$ is the number of HUs active at time point $t$ (Figure $\mathrm{S3} \mathrm{A}$ ). This property is a consequence of the 
sparse weight matrix $\mathbf{W}$, indicated by its heavy-tail log-distribution (Figure 2I, purple). The compositional phase is the norm for the presently estimated cRBMs, evidenced by the distribution of median $m(t)$ values for all recordings (average $\frac{\operatorname{median}(m)}{M}$ is 0.26 , see Figure $\mathrm{S} 3 \mathrm{~B}$ ). Importantly, the sparse weight matrix does not automatically imply that only a small subset of neurons is connected to the cRBM hidden layer. We validated this by observing that more neurons strongly connect to the hidden layer than expected by shuffling the weight matrix (Figure 2J).

Sparsity ensures that each assembly only connects to a handful of anatomical regions, as we quantified by calculating the overlap between cRBM assemblies and anatomical regions (Figure S4). We found that cRBM assemblies connect to a median of 3 regions (interquartile range: 2 to 6 regions). Importantly, the cRBM has no information about the locations of neurons during training, so the localization to a limited set of anatomical areas that we observe is extracted from the neural co-activation properties alone. For comparison, Principal Component Analysis (PCA), a commonly used non-sparse dimensionality reduction method that shares the cRBM architecture, naturally converged to a non-sparse weight matrix (Figure21, yellow), with fewer connected neurons than expected by shuffling its weight matrix (Figure $2 \mathrm{~K}$ ). This led to unspecific assemblies that are difficult to interpret by anatomy (Figure S4). As a result, sparsity, a cRBM property shared with some other dimensionality reduction techniques (see Tubiana et al. (2019a) for an extensive comparison), is crucial to interpret the assemblies by anatomy as we demonstrate in the next section.

\section{3 cRBM assemblies compose functional circuits and anatomical structures}

Above, we have shown that cRBMs converge to sparse weight matrix solutions. This property enables us to visualize the neural assemblies as the collection of significantly connected neurons to an HU. Neurons from a given neural assembly display concerted dynamics, and so one may expect their spatial organization to reflect the neuroanatomy and functional organization of the brain. We here highlight a selection of salient examples of neural assemblies, illustrating that assemblies match well with anatomical structures and functional circuits, while the complete set of neural assemblies is presented in Supplementary Video 1. In particular, we identified assemblies that together compose a neural circuit, are neurotransmitter-specific, encompass a long-range pathway, or can be identified by anatomy. The examples shown here are from a single fish (\#3), but results from other fish were comparable.

First, we identified six assemblies that together span the hindbrain circuit that drives eye and tail movements (Dunn et al. 2016; Wolf et al., 2017; Chen et al., 2018). We find two neural assemblies in rhombomere 2 which align with the anterior rhombencephalic turning region (ARTR, (Ahrens et al., 2013; Dunn et al., 2016; Wolf et al., 2017), Figure 3A-B). Each assembly primarily comprises neurons of either the left or right side of the ARTR, but also includes a small subset of contralateral neurons with weights of opposite sign in line with the established mutual inhibition between both subpopulations. Two other symmetric assemblies (Figure 3 C, D) together encompass the oculomotor nucleus (nIII) and the contralateral abducens nucleus (nVI, in rhombomere 6), two regions engaged in ocular saccades (Ma et al., 2014) and under the control of the ARTR (Wolf et al., 2017). Additionally, we observed two symmetric assemblies (Figure $3 \mathrm{E}, \mathrm{F}$ ) in the posterior hindbrain (in rhombomere 7), in a region known to drive unilateral tail movements (Chen et al., 2018; Marques et al., 2020) and whose antiphasic activation is also controlled by the ARTR activity (Dunn et al. (2016)).

Next, we observed assemblies that correspond to particular neurotransmitter expressions, such as the excitatory Vglut2 (Figure 3 $\mathrm{G}$ ) and inhibitory Gad1b (Figure $3 \mathrm{H}$ ) neurotransmitters. These assemblies consist multiple dense loci that sparsely populate the entire brain, confirming that cRBMs are able to capture a large morphological diversity of neural assemblies. Figure 3I depicts another sparse, brain-wide assembly that encompasses the pallium, habenula ( $\mathrm{Hb}$ ) and interpeduncular nucleus (IPN), and thus captures the Hb-IPN pathway that connects to other regions such as the pallium (Beretta et al., 2012).

Larger nuclei or circuits were often composed of a small number of distinct neural assemblies with some overlap. For example, the cerebellum was decomposed into multiple, bilateral assemblies (Figure 3J) whereas neurons in the torus semicircularis were grouped per brain hemisphere (Figure $3 \mathrm{~K}$ ). As a last example, the optic 


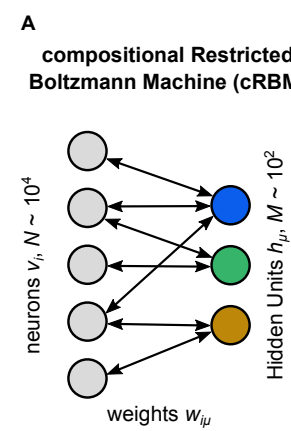

B

cRBM data generation schematic

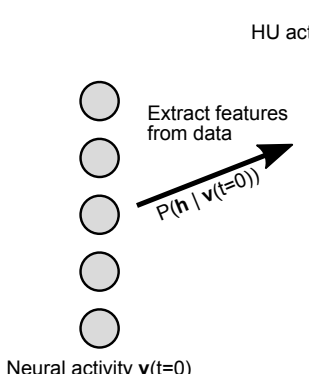

$\mathrm{HU}$ activity $\mathbf{h}(\mathrm{t}=0)$

Neural activity $\mathbf{v}(\mathrm{t}=0)$

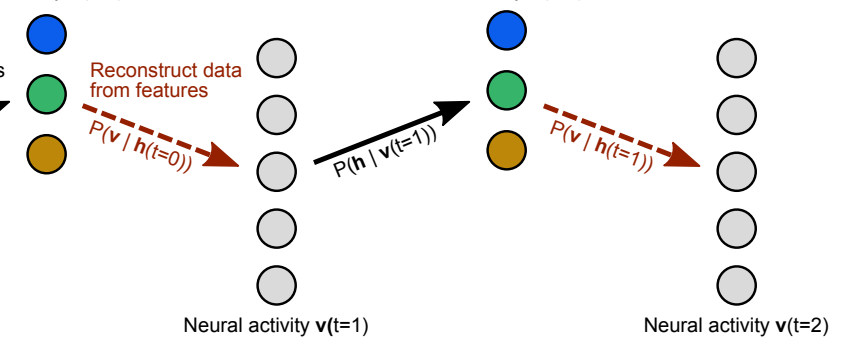

Sampling steps
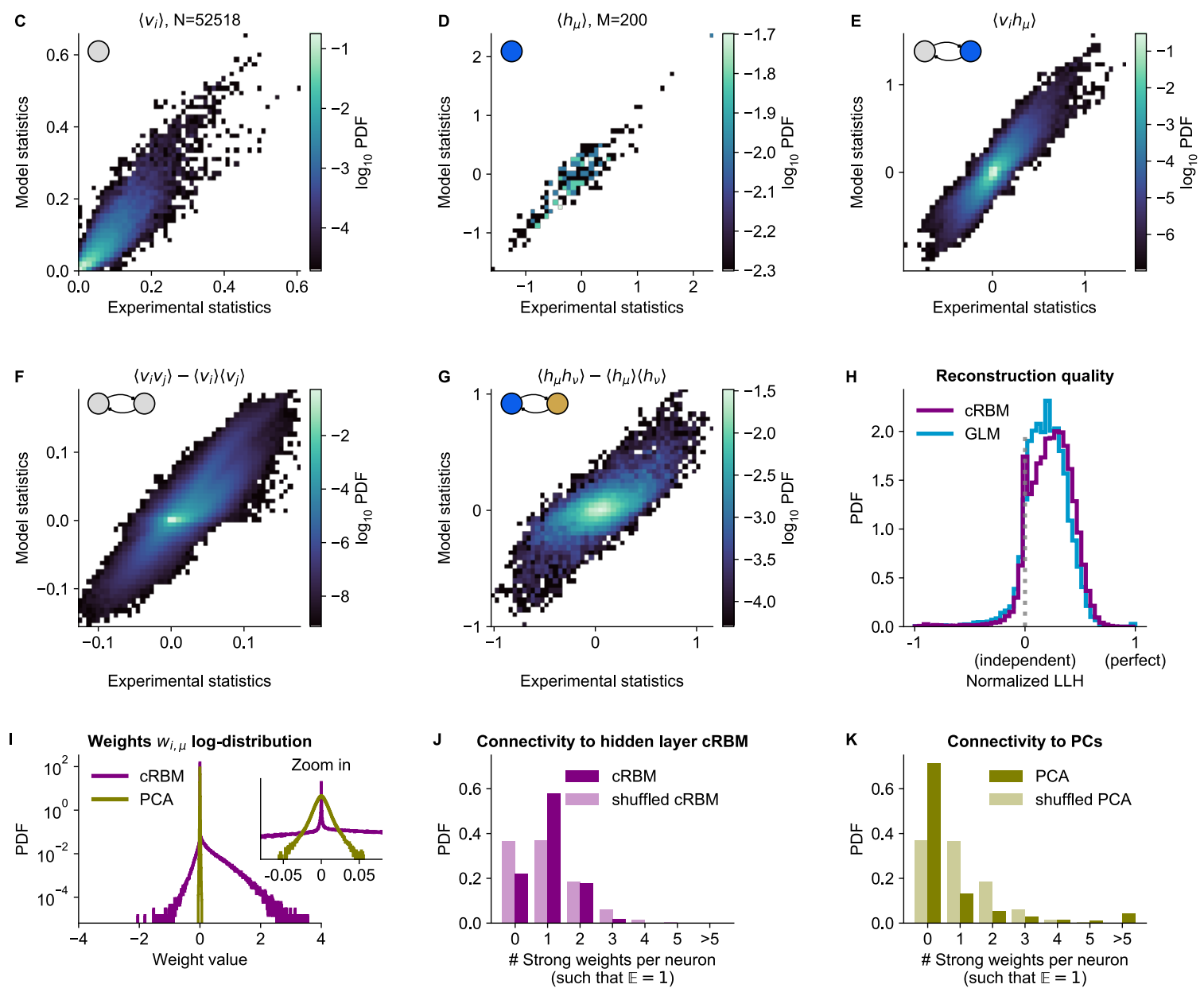

Figure 2: (Legend on next page)

tectum was composed of a larger set of approximately 18 neural assemblies, which spatially tiled the volume of the optic tectum (Figure 3L). This particular organization is suggestive of spatially localized interactions within

the optic tectum, and aligns with the morphology of previously inferred assemblies in this specific region (Romano et al. 2015: Diana et al. 2019; Triplett et al., 2020). However, Figure 3 altogether demonstrates that the typical assembly morphology of the optic tectum identified by our and these previous analyses does not readily 
Figure 2: cRBM is optimized to accurately replicate data statistics

(A) Schematic of the cRBM architecture, with neurons $v_{i}$ on the left, HUs $h_{\mu}$ on the right, connected by weights $w_{i, \mu}$. (B) Schematic depicting how cRBMs generate new data. The HU activity $\mathbf{h}(t)$ is sampled from the visible unit (i.e. neuron) configuration $\mathbf{v}(t)$, after which the new visible unit configuration $\mathbf{v}(t+1)$ is sampled and so forth.

(C) cRBM-predicted and experimental mean neural activity $\left\langle v_{i}\right\rangle$ were highly correlated (Pearson correlation $r_{\mathrm{P}}=0.91, P<10^{-307}$ ) and had low error (nRMSE $\left\langle_{\left.v_{i}\right\rangle}=0.11\right.$, normalized Root Mean Square Error, see STAR methods). Data displayed as 2D probability density function (PDF), scaled logarithmically (base 10).

(D) cRBM-predicted and experimental mean Hidden Unit (HU) activity $\left\langle h_{\mu}\right\rangle$ also correlated very strongly $\left(r_{\mathrm{P}}=0.93, P<10^{-86}\right)$ and had low nRMSE $\left\langle h_{\mu}\right\rangle=0.15$ (other details as in C)

(E) cRBM-predicted and experimental average pairwise neuron-HU interactions $\left\langle v_{i} h_{\mu}\right\rangle$ correlated strongly $\left(r_{\mathrm{P}}=0.74, P<10^{-307}\right)$ and had a low error $\left(\mathrm{nRMSE}_{\left\langle v_{i} h_{\mu}\right\rangle}=0.09\right)$.

(F) cRBM-predicted and experimental average pairwise neuron-neuron interactions $\left\langle v_{i} v_{j}\right\rangle$ correlated well $\left(r_{\mathrm{P}}=0.58, P<10^{-307}\right)$ and had a low error $\left(\mathrm{nRMSE}_{\left\langle v_{i} v_{j}\right\rangle}=-0.09\right.$, where the negative nRMSE value means that $\mathrm{cRBM}$-predictions match the test data slightly better than the train data). Pairwise interactions were corrected for naive correlations due to their mean activity by subtracting $\left\langle v_{i}\right\rangle\left\langle v_{j}\right\rangle$.

(G) cRBM-predicted and experimental average pairwise HU-HU interactions $\left\langle h_{\mu} h_{v}\right\rangle$ correlated strongly $\left(r_{\mathrm{P}}=0.73, P<10^{-307}\right)$ and had a low error (nRMSE $\left.\left\langle h_{\mu} h_{v}\right\rangle=0.17\right)$.

(H) The low-dimensional cRBM bottleneck reconstructs most neurons above chance level (purple), quantified by the normalized loglikelihood (nLLH) between neural test data $v_{i}$ and the reconstruction after being transformed to HU activity (see STAR methods). Median normalized $\mathrm{nLLH}_{\mathrm{CRBM}}=0.24$. Reconstruction quality was also determined for a fully connected Generalized Linear Model (GLM) that attempted to reconstruct the activity of a neuron $v_{i}$ using all other neurons $\mathbf{v}_{-i}$ (see STAR Methods). The distribution of 5000 randomly chosen neurons is shown (blue), with median $\mathrm{nLLH}_{\mathrm{GLM}}=0.20$. The $\mathrm{cRBM}$ distribution is stochastically greater than the GLM distribution (one-sided Mann Whitney U test, $P<10^{-42}$ ).

(I) cRBM (purple) had a sparse weight distribution, but exhibited a greater proportion of large weights $w_{i, \mu}$ than PCA (yellow), both for positive and negative weights, displayed in log-probability.

(J) Distribution of above-threshold absolute weights $\left|w_{i, \mu}\right|$ per neuron $v_{i}$ (dark purple), indicating that more neurons strongly connect to the cRBM hidden layer than expected by shuffling the weight matrix of the same cRBM (light purple). The threshold $\Theta$ was set such that the expected number of above-threshold weights per neuron $\mathbb{E}\left(\# \mathbf{w}_{i}>\Theta\right)=1$.

(K) Corresponding distribution as in (J) for PCA (dark yellow) and its shuffled weight matrix (light yellow), indicating a predominance of small weights in PCA for most neurons $v_{i}$.

All panels of this figure show the data statistics of the cRBM with parameters $M=200$ and $\lambda=0.02$ (best choice after cross-validation, see Figure S1] of example fish \#3, comparing the experimental test data test and model-generated data after cRBM training converged.

generalize to other brain regions, where a large range of different assembly morphologies compose neural circuits.

Overall, the clear alignment of cRBM-based neural assemblies with anatomical regions and circuits suggests that cRBMs are able to identify anatomical structures from dynamical activity alone, which enables them to break down the overall activity into parts that are interpretable by physiologists in the context of previous, more local studies.

\subsection{HU dynamics cluster into groups and display slower dynamics than neurons}

HU activity, defined as the expected value of $P(\mathbf{h} \mid \mathbf{v})$ (Equation 9), exhibits a rich variety of dynamical patterns (Figure 4A). HUs can activate very transiently, slowly modulate their activity, or display periods of active and inactive states of comparable duration. Figure $4 \mathrm{~B}$ highlights a few $\mathrm{HU}$ activity traces that illustrate this diversity of HU dynamics. The top three panels of Figure $4 \mathrm{~B}$ show the dynamics of the assemblies of Figure 3 A-F which encompass the ARTR hindbrain circuit that controls saccadic eye movements and directional tail flips. HUs 99 and 161 drive the left and right ARTR and display antiphasic activity with long dwell times of $\sim 15 \mathrm{~s}$, in accordance with previous studies (Ahrens et al., 2013, Dunn et al., 2016, Wolf et al., 2017). HU 102 and 163 correspond to the oculomotor neurons in the nuclei nIII and nVI that together drive the horizontal saccades. Their temporal dynamics are locked to that of the ARTR units in line with the previously identified role ARTR as a pacemaker for the eye saccades (Wolf et al., 2017). HUs 95 and 135, which drive directional tail flips, display transient activations that only occur when the ipsilateral ARTR-associated HU is active. This is consistent with the previous finding that the ARTR alternating activation pattern sets the orientation of successive tail flips accordingly (Dunn et al. 2016). The fourth panel shows the traces of the brain-wide assemblies of Figures $3 \mathrm{G}$, I, displaying slow tonic modulation of their activity. Finally, the bottom panel, that corresponds to the collective dynamics of assembly 122 (Figure $3 \mathrm{H}$ ) comprises short transient activity that likely corresponds 
bioRxiv preprint doi: https://doi.org/10.1101/2021.11.09.467900; this version posted November 11, 2021. The copyright holder for this preprint (which was not certified by peer review) is the author/funder, who has granted bioRxiv a license to display the preprint in perpetuity. It is made available under aCC-BY-NC-ND 4.0 International license.

Neural circuit composed of assemblies
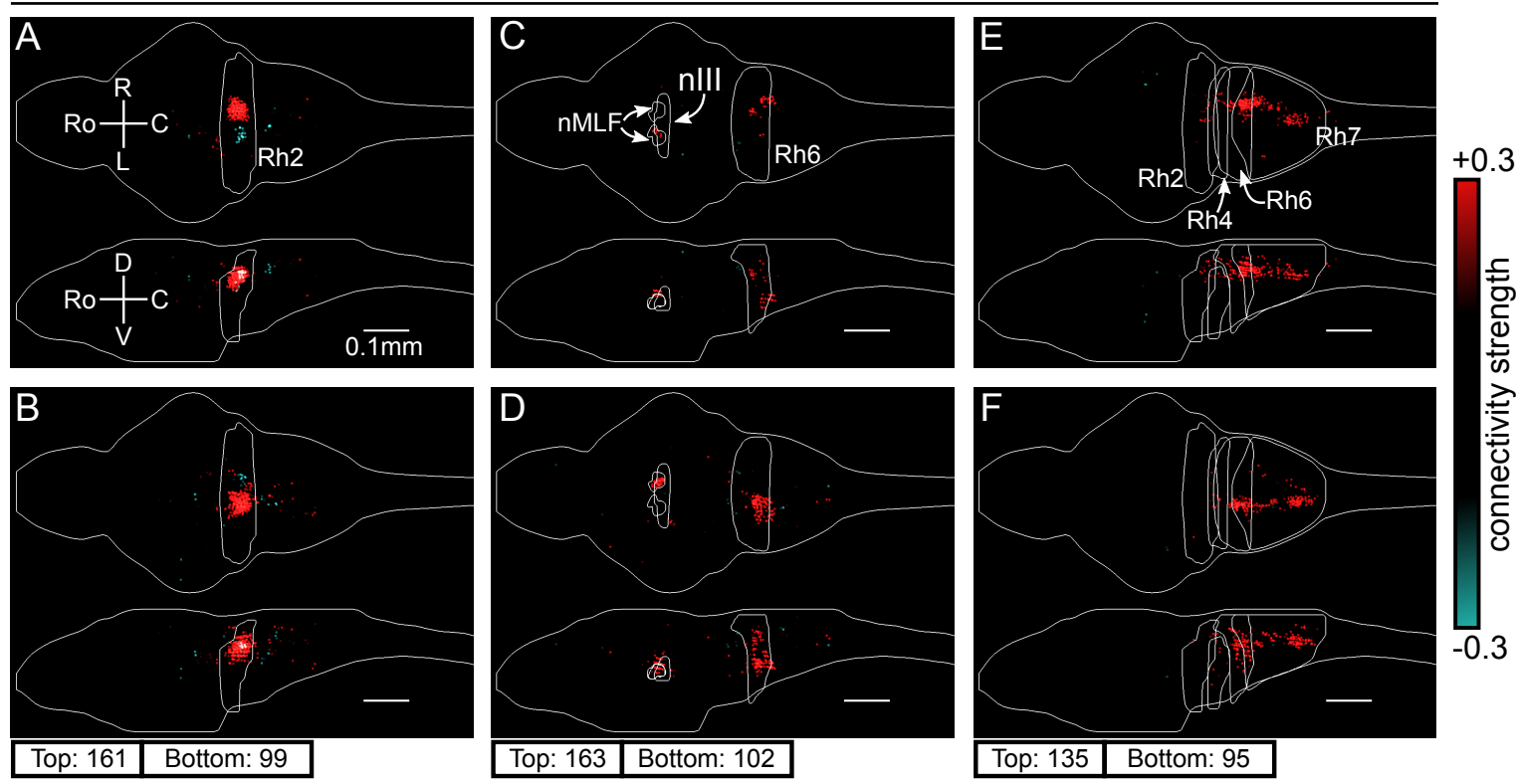

Neurotransmitter-specific assemblies
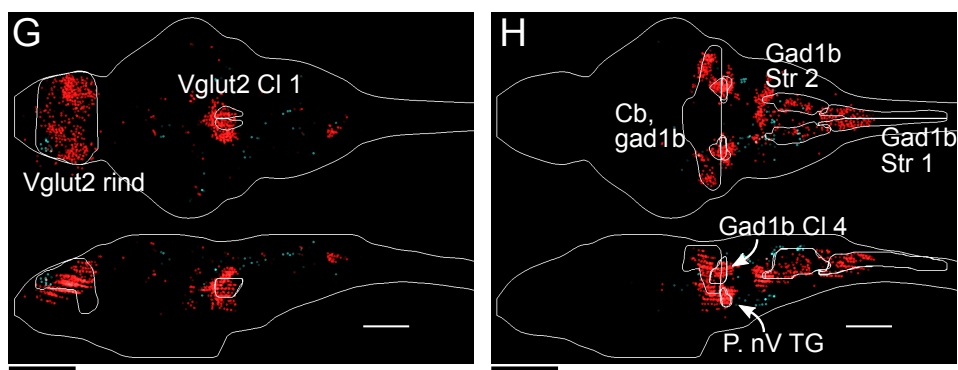

Pathway assembly

178
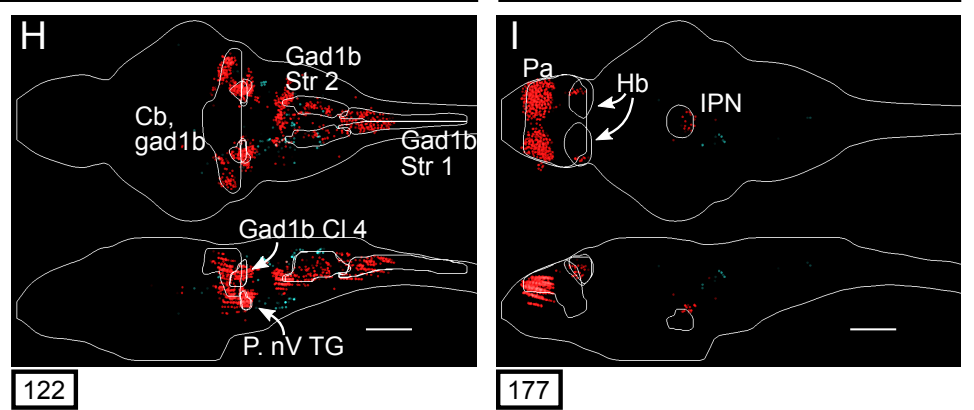

177

Groups of assemblies identified by anatomy
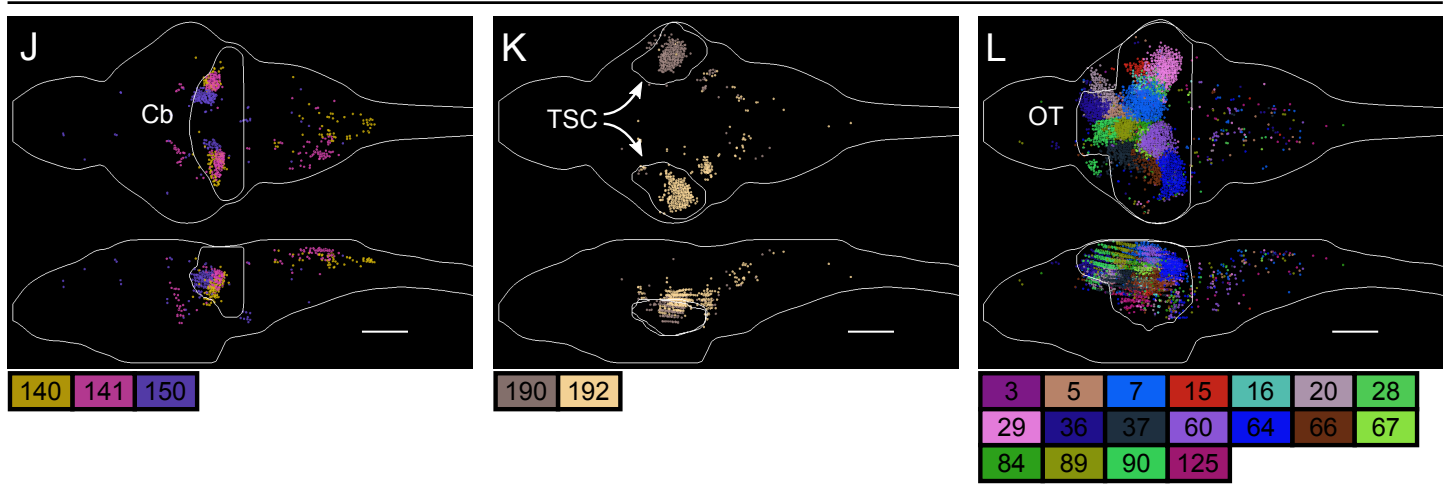

Figure 3: (Legend on next page)

to fictive swimming events.

Some HUs regularly co-activate, leading to strong correlations between different HUs. This is quantified by 
Figure 3: cRBM assemblies compose functional circuits and anatomical structures

(A)-(I) Individual example assemblies $\mu$ are shown by coloring each neuron $i$ with its connectivity weight value $w_{i, \mu}$ (see color bar at the right hand side). The assembly index $\mu$ is stated at the bottom of each panel. The orientation and scale are given in panel A (Ro: rostral, C: caudal, R: right, L: left, D: dorsal, V: ventral). Anatomical regions of interest, defined by the ZBrain Atlas (Randlett et al. 2015), are shown in each panel (Rh: rhombomere, nMLF: nucleus of the medial longitudinal fascicle; nIII: oculomotor nucleus nIII, Cl: cluster; Str: stripe, P. nV TG: Posterior cluster of nV trigeminal motorneurons; Pa: pallium; Hb: habenula; IPN: interpeduncular nucleus).

(J)-(L) Groups of example assemblies that lie in the same anatomical region are shown for cerebellum (Cb), torus semicircularis (TSC) and optic tectum (OT). Neurons $i$ were defined to be in an assembly $\mu$ when $\left|w_{i, \mu}\right|>0.15$, and colored accordingly. If neurons were in multiple assemblies shown, they were colored according to their strongest-connecting assembly.

their Pearson correlation matrix shown in Figure $4 \mathrm{C}$ (top), which reveals clusters of correlated HUs. These were grouped using hierarchical clustering (Figure $4 \mathrm{C}$, bottom), and we then manually identified their main anatomical location (top labels). We further observed that $\mathrm{HU}$ activity is bimodal, as evidenced by the distribution of all HU activity traces in Figure 4D. This bimodality can emerge because the dReLU potentials $\mathcal{U}_{\mu}$ (Equation 14) can learn to take different shapes, including a double-well potential that leads to bimodal dynamics (see STAR Methods). This allows us to effectively describe HU activity as a two-state system, where $h_{\mu}(t)>0$ increases the probability to spike $\left(P\left(v_{i}(t)=1\right)\right)$ for its positively connected neurons, and $h_{\mu}(t)<0$ decreases their probability to spike. The binarized neuron activity is also a two-state system (spiking or not spiking), which enabled us to compare the time constants of neuron and HU state changes, quantified by the median time between successive onsets of activity. We find that HUs, which represent the concerted dynamics of neuronal assemblies, operate on a slower time scale than individual neurons (Figure $4 \mathrm{E}$ ). This observation aligns with the expected difference between cellular and circuit-level time scales.

\section{5 cRBM embodies functional connectivity that is strongly correlated across individ- uals}

The probabilistic nature of cRBMs uniquely enables a direct and theoretically grounded estimation of the functional connection $J_{i j}$ between pairs of neurons, where $J_{i j}$ quantifies the direct impact of the state of neuron $j$ on the probability to spike of neuron $i$. We first defined the generic, symmetric functional connection $J_{i j}$ using $P\left(v_{i} \mid v_{j}, v_{k \neq i, j}\right)$ (Equation 16) and then used $P(\mathbf{v})$ (Equation 12) to derive the cRBM-specific $J_{i j}$ (Equation 18 , see STAR Methods). Using this definition of $J_{i j}$, we constructed a full neuron-to-neuron effective connectivity matrix for each zebrafish recording. We then asked whether this cRBM-inferred connectivity matrix was robust across individuals. For this purpose we calculated the functional connections between anatomical regions, given by the assemblies that occupy each region, because neuronal identities can vary across individual specimen. For this purpose we considered anatomical regions as defined by the mapzebrain atlas (Kunst et al., 2019) for which a regional-scale structural connectivity matrix exists to which we will compare our functional connectivity matrix.

We aggregated neurons using the $L_{1}$ norm for each pair of anatomical regions to determine the functional connection between regions (see STAR Methods). This led to a symmetrical functional connectivity matrix for each animal, three of which are shown in Figure 5 A-C (where non-imaged regions are left blank, and all 8 animals are shown in Figure S5]. The strength of functional connections is distributed approximately lognormal (Figure 5D), similar to the distribution of structural region-to-region connections (Kunst et al., 2019). To quantify the similarity between individual fish, we computed the Pearson correlation between each pair of fish. Functional connectivity matrices correlate strongly across individuals, with an average Pearson correlation of 0.69 (Figures $5 \mathrm{E}$ and $\mathrm{F}$ ).

We conclude that similar functional circuits spontaneously activate across individuals, despite the limited duration of neural recordings ( $\sim 25$ minutes), which can be identified across fish using independently estimated cRBMs. In the next section we aggregate these individual matrices to a general functional connectivity matrix for comparison with the zebrafish structural connectivity matrix. 

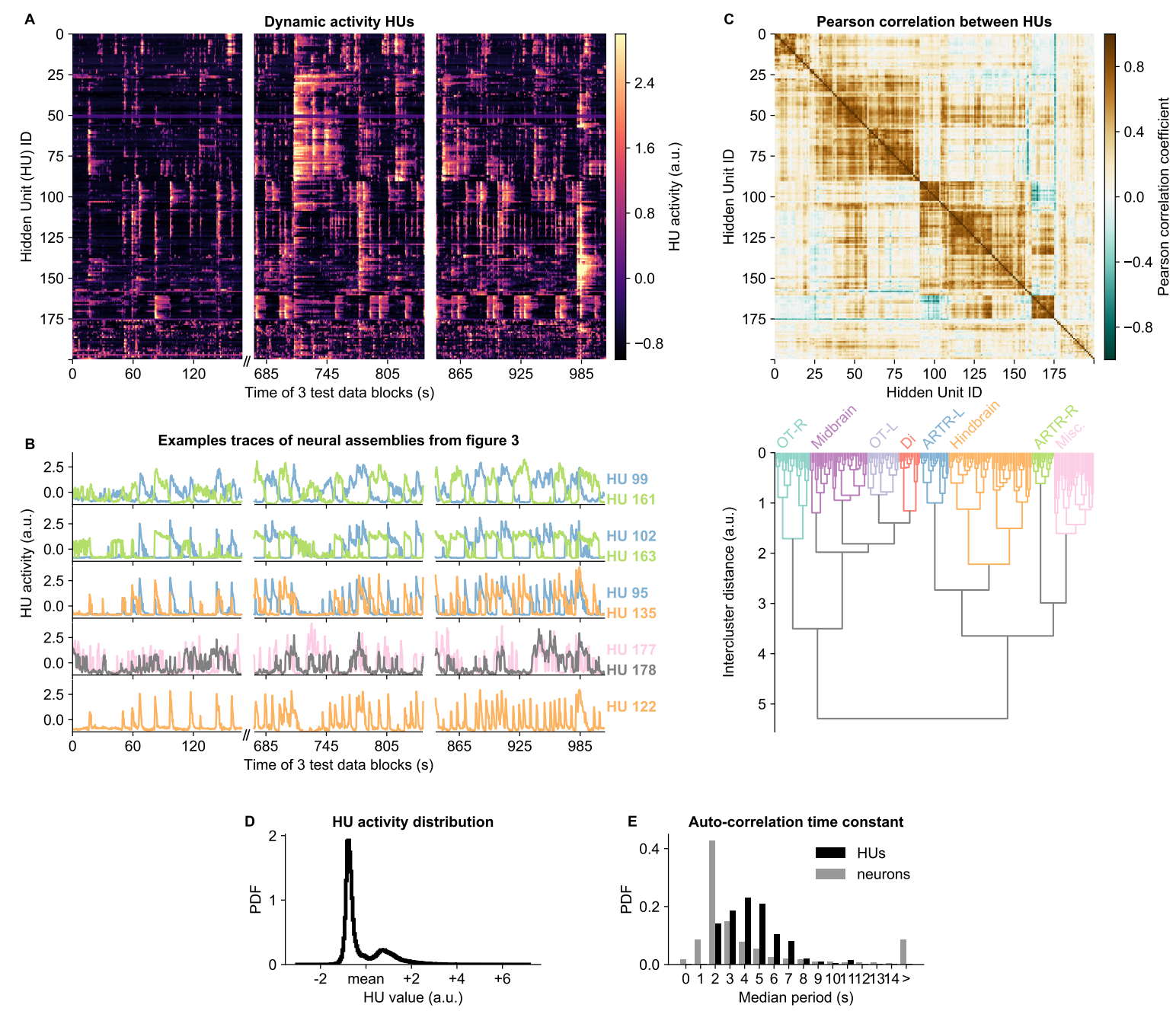

Figure 4: HU dynamics are bimodal and activate slower than neurons

(A) HU dynamics are diverse and are partially shared across HUs. The bimodality transition point of each HU was determined and subtracted individually, such that positive values correspond to HU activation (see STAR methods). The test data consisted of 3 blocks, with a discontinuity in time between the first and second block (STAR methods).

(B) Highlighted example traces from panel A. HU indices are denoted on the right of each trace, colored according to their cluster from panel D. The corresponding cellular assemblies of these HU are shown in Figure 3 A-I.

(C) Top: Pearson correlation matrix of the dynamic activity of panel A. Bottom: Hierarchical clustering of the Pearson correlation matrix. Clusters (as defined by the colors) were annotated manually. This sorting of HUs is maintained throughout the manuscript. OT: Optic Tectum, Di: Diencephalon, ARTR: anterior rhombencephalic turning region, Misc.: Miscellaneous, L: Left, R: Right.

(D) The distribution of all HU activity values of panel A shows that HU activity is bimodal. PDF: Probability Density Function.

(E) Distribution of the time constants of HUs (black) and neurons (grey). Time constants are defined as the median oscillation period, for both HUs and neurons. An HU oscillation is defined as a consecutive negative and positive activity interval. A neuron oscillation is defined as a consecutive interspike-interval and spike-interval (which can last for multiple time steps, for example see Figure 1 A). The time constant distribution of HUs is greater than the neuron distribution (Mann Whitney $\mathrm{U}$ test, $P<10^{-16}$ ).

\section{6 cRBM-inferred functional connectivity reflects structural connectivity}

In the previous section we have determined the functional connections between anatomical regions using the cRBM assembly organization. Although functional connectivity stems from the structural (i.e. biophysical) connections between neurons, it can reflect correlations that arise through indirect network interactions (Bassett) \& Sporns, 2017, Das \& Fiete, 2020). Using recently published structural connectivity data of the zebrafish brain (Kunst et al. 2019), we are now able to quantify the overlap between a structurally defined connectivity 

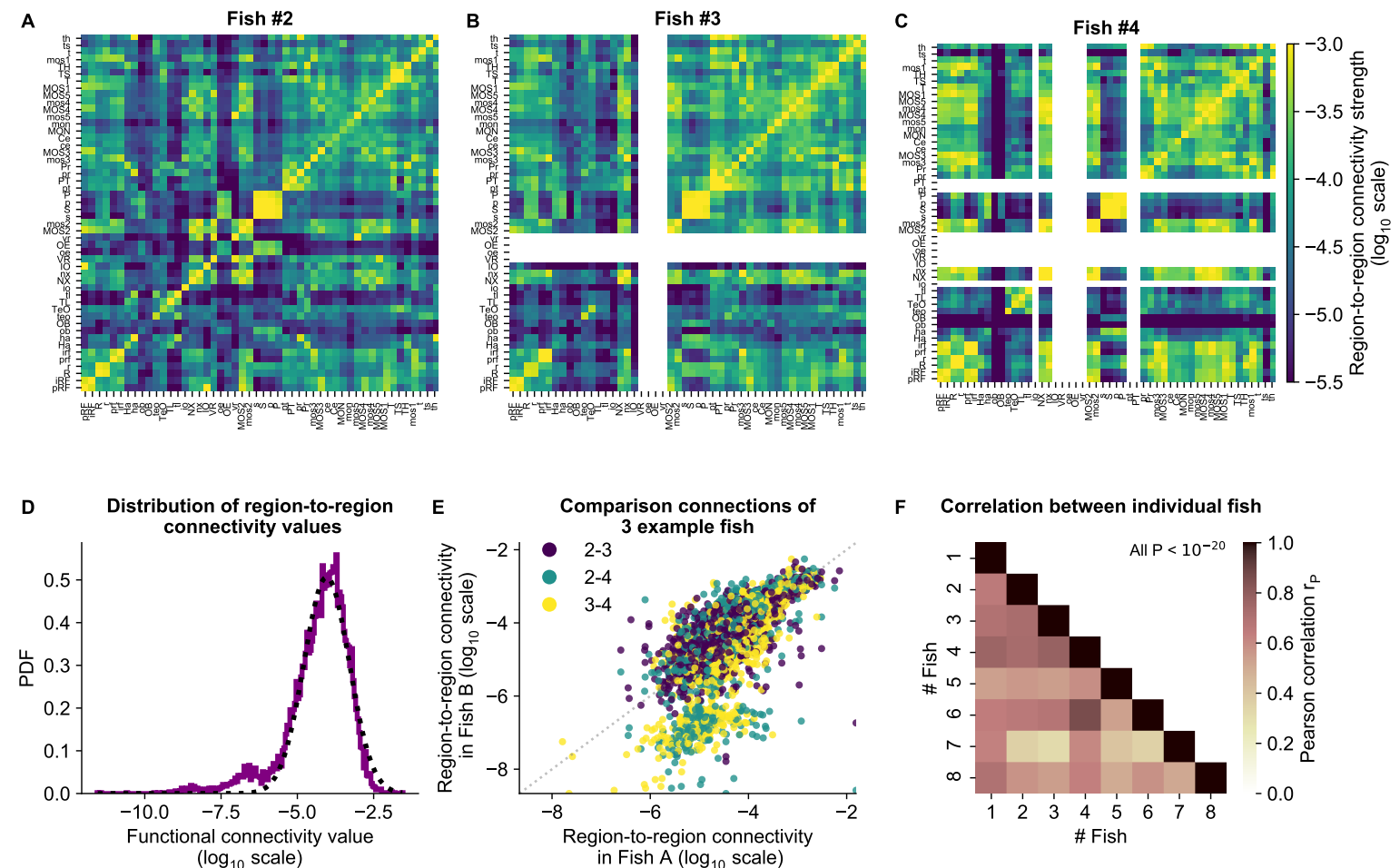

Figure 5: cRBM gives rise to functional connectivity that is strongly correlated across individuals

(A) The functional connectivity matrix between anatomical regions of the mapzebrain atlas (Kunst et al. 2019) of example fish \#2 is shown. Functional connections between two anatomical regions were determined by the similarity of the HUs to which neurons from both regions connect to (STAR methods). Mapzebrain atlas regions with less than 5 imaged neurons were excluded, yielding $N_{\text {MAP }}=50$ regions in total. See Table $\mathrm{S} 1$ for region name abbreviations. The matrix is shown in $\log _{10}$ scale, because functional connections are distributed approximately log-normal (see panel D).

(B) Equivalent figure for example fish \#3 (example fish of prior figures).

(C) Equivalent figure for example fish \#4. Panels A-C share the same $\log _{10}$ color scale (right).

(D) Functional connections are distributed approximately log-normal. (Mutual information with a log-normal fit (black dashed line) is 3.83 , while the mutual information with a normal fit is 0.13 ). All connections of all 8 fish are shown, in $\log _{10}$ scale (purple).

(E) Functional connections of different fish correlate well, exemplified by the 3 example fish of panels A-C. All non-zero functional connections (x-axis and y-axis) are shown, in $\log _{10}$ scale. Pearson correlation $r_{\mathrm{P}}$ between pairs: $r_{\mathrm{P}}(\# 2, \# 3)=0.73, r_{\mathrm{P}}(\# 2, \# 4)=0.73$, $r_{\mathrm{P}}(\# 3, \# 4)=0.78$. All correlation $\mathrm{P}$ values $<10^{-20}$.

(F) Pearson correlations $r_{\mathrm{P}}$ of region-to-region functional connections between all pairs of 8 fish. For each pair, regions with less than 5 neurons in either fish were excluded. All $\mathrm{P}$ values $<10^{-20}$, and average correlation value is 0.69 .

matrix and our functional connectivity matrix estimated through neural dynamics. Kunst et al. (2019) deter-

med a zebrafish structural connectivity matrix between 72 anatomical regions using structural imaging data from thousands of individually Green Fluorescent Protein (GFP)-labeled neurons from multiple animals. We slightly extended this matrix by using the most recent data, filtering indirect connections and accounting for the resulting sampling bias (Figure 6A, regions that were not imaged in our light-sheet microscopy experiments were excluded). Next, we aggregated the functional connectivity matrices of all our calcium imaging recordings to one grand average functional connectivity matrix (Figure 6 $\mathrm{B}$ ).

For comparison, we also calculated the connectivity matrices defined by either covariance or Pearson correlation (Figure S6). The cRBM functional connectivity spans a larger range of values than either of these methods, leading to a more fine-grained connectivity matrix akin to the structural connectivity map (Figure 6B).

This greater visual resemblance was statistically confirmed by calculating the Spearman correlation between structural and functional connectivity, which is greater for cRBM $\left(r_{\mathrm{S}}=0.39\right.$, Figure $\left.6 \mathrm{C}\right)$, than for covariance- 
bioRxiv preprint doi: https://doi.org/10.1101/2021.11.09.467900; this version posted November 11,2021 . The copyright holder for this preprint (which was not certified by peer review) is the author/funder, who has granted bioRxiv a license to display the preprint in perpetuity. It is made available under aCC-BY-NC-ND 4.0 International license.

based connectivity $\left(r_{\mathrm{S}}=0.18\right.$, Figure $\mathrm{S} 6$ left $)$ or correlation-based connectivity $\left(r_{\mathrm{S}}=0.26\right.$, Figure $\mathrm{S} 6$ ] right $)$. Hence, using recordings of $\sim 25$ minutes on average, cRBMs were able to identify functional connections that resemble the anatomical connectivity between brain regions. Strong or weak functional connections are predictive of present or absent structural connections respectively (Figure 6D), and could thus potentially be used for inference in systems where the structural connectivity pattern is unknown.
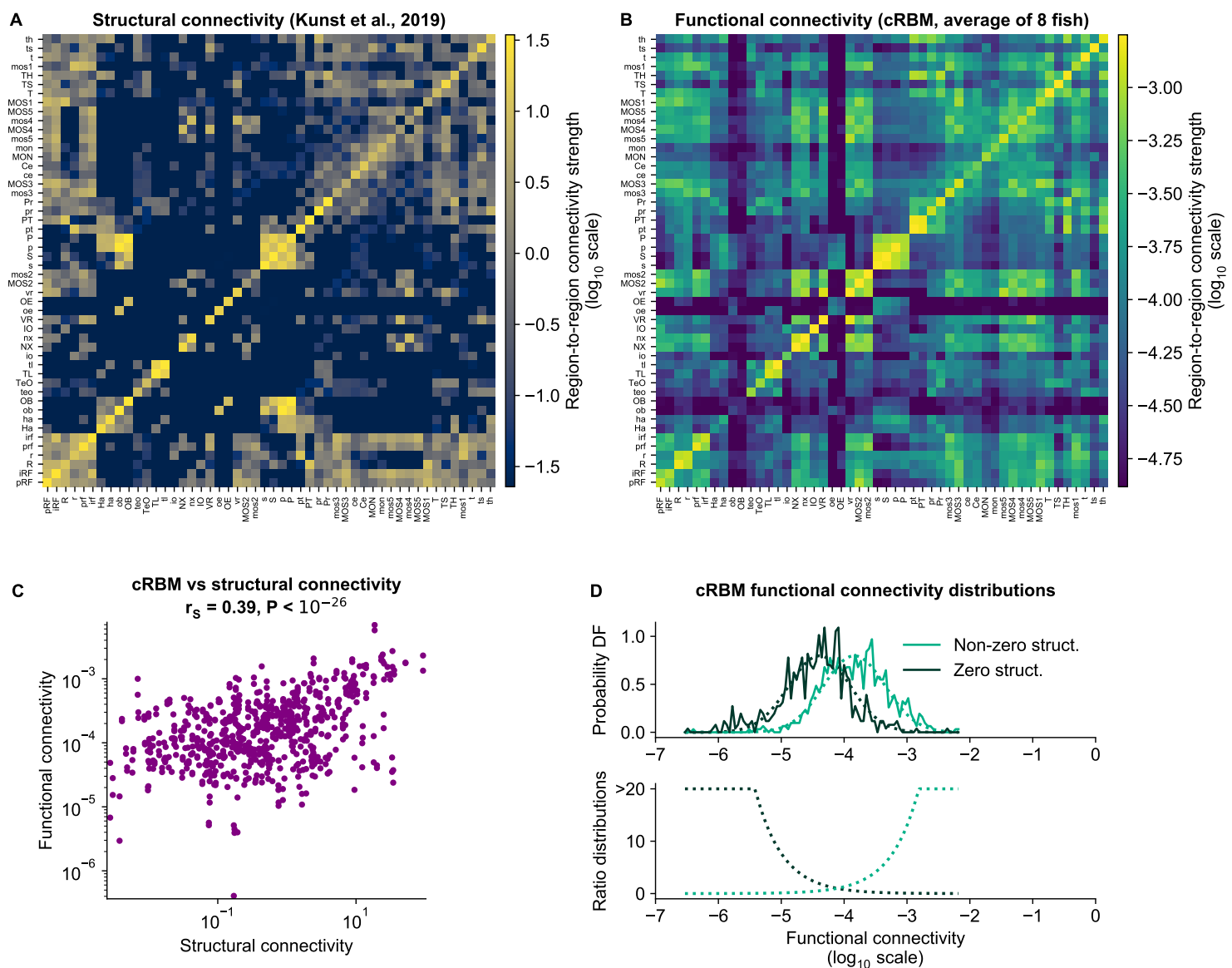

Figure 6: cRBM-inferred functional connectivity reflects structural connectivity

(A) Structural connectivity matrix is shown in $\log _{10}$ scale, adapted from Kunst et al. (2019). Regions that were not imaged in our experiments were excluded (such that $N_{\mathrm{MAP}}=50$ out of 72 regions remain). Regions (x-axis and y-axis) were sorted according to Kunst et al. [2019]. Additional structural data was added and the normalization procedure was updated to include within-region connectivity (see STAR methods). See Table S1 for region name abbreviations.

(B) Average functional connectivity matrix is shown in $\log _{10}$ scale, as determined by averaging the $\mathrm{cRBM}$ functional connectivity matrices of all 8 fish (see STAR methods). The same regions (x-axis and y-axis) are shown as in panel A.

(C) Functional and structural connectivity of panels A and B correlate well, with Spearman correlation $r_{\mathrm{S}}=0.39$. Each data point corresponds to one region-to-region pair. Data points for which the structural connection was exactly 0 were excluded (see panel D for their analysis).

(D) The distribution of functional connections of region pairs with non-zero structural connections is greater than functional connections corresponding to region pairs without structural connections $\left(P<10^{-15}\right.$, two-sided Kolmogorov-Smirnov test). The bottom panel shows the evidence for inferring either non-zero or zero structural connections, defined as the fraction between the PDFs of the top panel (fitted Gaussian distributions were used for denoising). 


\section{Discussion}

We have developed a cRBM model that accurately replicated the data statistics of brain-scale zebrafish recordings, thereby forming neural assemblies that spanned the entire brain. The objective of our study was threefold: first, to show that the cRBM model can be applied to high-dimensional data, such as whole-brain recordings, second, to prove that an assembly-based model is sufficient to generate whole-brain neural data statistics, and third, to describe the physiological properties of the assembly organization in the zebrafish brain and use it to create a functional connectivity map. We have shown that, after convergence, the cRBM-generated data not only replicated the data statistics that it was constrained to fit, but also extrapolated to fit the pairwise correlation statistics of neurons and HUs, leading to a better reconstruction of neural data than a fully connected GLM (Figure 2). These results thereby quantify how neural assemblies play a major role in determining the collective dynamics of the brain. To achieve this, cRBMs formed sparsely localized assemblies that spanned the entire brain, facilitating their biological interpretation (Figure 3, S4, 4). Further, the probabilistic nature of the cRBM model allowed us to create a mesoscale functional connectivity map that was largely conserved across individual fish and correlated well with structural connectivity (Figures 5, 6,

The maximum entropy principle underlying the cRBM definition has been a popular method for inferring pairwise effective connections between neurons or assemblies of co-activating cells (Schneidman et al., 2006; Tavoni et al., 2017, Ferrari et al., 2017, Meshulam et al., 2017; Posani et al., 2018; Chen et al., 2019). However, its computational cost has limited this pairwise connectivity analysis to typically $\sim 10^{2}$ neurons. The two-layer cRBM model that we used here alleviates this burden, because the large number of neuron-to-neuron connections are no longer explicitly optimized, which enables a fast data sampling procedure (Figure $2 \mathrm{~B}$ ). However, we have shown that these connections are still estimated indirectly with high accuracy via the assemblies they connect to (Figure $2 \mathrm{~F}$ ). We have thus shown that the cRBM is able to infer the $\frac{1}{2} N^{2} \approx 10^{9}$ (symmetric) pairwise connections through its assembly structure, a feat that is computationally infeasible for many other methods.

Previously, we have extensively compared cRBM performance to other dimensionality reduction techniques, including Principal Component Analysis (PCA), Independent Component Analysis (ICA), Variational Autoencoders (VAEs) and their sparse variants, using protein sequence data as a benchmark (Tubiana et al., 2019a). nature, putting too much emphasis on low-probability high-variance states, while VAEs were unable to capture all features of data due to the unrealistic assumption of independent, Gaussian-distributed latent variables. Additionally, while PCA has previously been successful in describing zebrafish neural dynamics in terms of their main covariances modes (Ahrens et al. 2012; Marques et al., 2020), we show here that it is not appropriate for assembly extraction due to the absence of both a compositional and stochastic nature (Figures 2, S4). Furthermore, we have shown that the generative component of cRBM models is essential for quantitatively assessing that the assembly organization is sufficient for reproducing neural statistics (Figure 2), moving beyond deterministic clustering analyses such as k-means (Panier et al. 2013, Chen et al. 2018), similarity graph clustering (Mölter et al. 2018) or non-negative matrix factorization (Mu et al. 2019).

After having quantitatively validated the resultant assemblies, we moved to discussing the biological implications of our findings. Previous studies of the zebrafish optic tectum have identified neural assemblies that were spatially organized into single dense clusters of cells (Romano et al., 2015, Diana et al., 2019; Triplett et al. 2020). We have replicated these findings by observing the distinct organization of ball-shaped assemblies in the optic tectum (Figure 3L). However, our data extends to many other anatomical regions in the brain, where we found that assemblies can be much more dispersed, albeit still locally dense, consisting of multiple clusters of neurons (Figure 3 .

cRBM allowed us to compute the effective, functional connection between each pair of neurons, aggregated to functional connections between each pair of regions. Importantly, we found that this region-scale connectivity is well-conserved across specimen. This observation is non-trivial because each recording only lasted $\sim 25$ minutes, which represents a short trajectory across accessible brain states. It suggests that, although each 
individual brain may be unique at the neuronal scale, the functional organization could be highly stereotyped at a sufficiently coarse-grained level.

345

It would be naive to assume that these functional connections equate biophysical, structural connections (Das \& Fiete, 2020). Both represent different, yet interdependent aspects of the brain organization. Indeed, we found that structural connectivity is well-correlated to functional connectivity, confirming that functional links are tied to the structural blueprint of brain connectivity (Figure 6). Furthermore, strong (weak) functional connections are predictive of present (absent) structural connections between brain regions, though intermediate values are ambiguous.

It will be crucial to synergistically merge structural and dynamic information of the brain to truly comprehend brain-wide functioning (Bargmann \& Marder, 2013, Kopell et al., 2014). Small brain organisms are becoming an essential means to this end, providing access to a relatively large fraction of cells (Ahrens \& Engert, 2015). To generate new scientific insights it is thus essential to develop analytical methods that can scale with the rapidly growing size of both structural and dynamic data (Helmstaedter, 2015, Ahrens, 2019). In this study we have established that the cRBM can model high-dimensional data accurately, and that its application to zebrafish recordings was crucial to unveil their brain-scale assembly organization. In future studies, cRBMs could be used to generate artificial data whose statistics replicate those of the zebrafish brain. This opens up a new avenue for in silico ablation and perturbation studies with strong physiological footing, crucial for developing hypotheses for future experimental work (Jazayeri \& Afraz, 2017, Das \& Fiete, 2020).

\section{Acknowledgements}

T.L.v.d.P. had an Erasmus+ fellowship (European Union) and acknowledges support from the Biotechnology and Biological Sciences Research Council (BBSRC, grant No. BB/M011224/1). J.T. acknowledges support from the Edmond J. Safra Center for Bioinformatics at Tel Aviv University and from the Human Frontier Science Program (cross-disciplinary postdoctoral fellowship LT001058/2019-C). G.M. was funded by a PhD fellowship from the Doctoral School in Physics, Ile de France (EDPIF). G.L.G. had a PhD fellowship from the Systems Biology Network of Sorbonne Université. B.E. and T.L.v.d.P. were supported by an NWO-VIDI Grant.

Funding sources: European Research Council (ERC) under the European Union's Horizon 2020 research and innovation program grant agreement number 715980. Human Frontier Science Program (RGP0060/2017). The French Research National Agency under grant No. ANR-16-CE16-0017. Dutch Institute for Scientific Research NWO (Nederlandse Organisatie voor Wetenschappelijk Onderzoek) grant No. 016.VIDI.189.052.

We thank the IBPS fish facility staff for the fish maintenance, in particular Stéphane Tronche and Alex Bois. We are grateful to Carounagarane Dore for his contribution to the design of the experimental setup. We thank Misha Ahrens for providing the GCaMP lines. We are also grateful to Rémi Monasson for very fruitful discussions and his comments on the manuscript.

\section{Author Contributions}

T.L.v.d.P., J.T., V.B., B.E. and G.D. conceived the project, analyzed data and wrote the manuscript. T.L.v.d.P. and J.T. developed the cRBM code, performed the various analyses and wrote the original draft. G.L.G. and G.M. carried out the functional neural recording experiments and associated data pre-processing. M.K. and H.B. produced the structural connectivity data. V.B., B.E. and G.D. supervised the work. All authors contributed to the writing and editing of the manuscript.

\section{Declaration of Interests}

The authors declare no competing interests. 


\section{STAR Methods}

\subsection{RESOURCE AVAILABILITY}

\subsubsection{Lead contact}

For further information and requests for resources and reagents, please contact the corresponding authors.

\subsubsection{Materials Availability}

Not applicable.

\subsubsection{Data and Code Availability}

The cRBM model has been developed in Python 3.7 and is available at: https://github.com/jertubiana/PGM

Calcium imaging data pre-processing was performed in MATLAB (Mathworks) using previously published protocols and software (Panier et al., 2013; Wolf et al., 2017; Migault et al., 2018; Tubiana et al., 2020). The functional data recordings and trained cRBM models will be made publicly available after publication.

Figures of neural assemblies or neurons (Figure 1, 3) were made using the Fishualizer, which is a 4D (space + time) data visualization software package that we have previously published (Migault et al., 2018), available at https://bitbucket.org/benglitz/fishualizer_public

Minor updates were implemented to tailor the Fishualizer for viewing assemblies, which can be found at https://bitbucket.org/benglitz/fishualizer_public/src/assembly_viewer

All other data analysis and visualization was performed in Python 3.7 using standard packages (numpy (Harris et al. 2020), scipy (Virtanen et al., 2020), scikit-learn (Pedregosa et al., 2011), matplotlib (Hunter. 2007), pandas (McKinney et al., 2010), seaborn (Waskom, 2021), h5py). The corresponding code is available

\subsection{EXPERIMENTAL MODEL AND SUBJECT DETAILS}

\subsubsection{Zebrafish larvae}

Experiments were conducted on nacre mutants, aged 5-7 days post-fertilization (dpf). Larvae were reared in Petri dishes at $28^{\circ} \mathrm{C}$ in embryo medium (E3) on a 14/10h light/dark cycle, and were fed powdered nursery food every day from $6 \mathrm{dpf}$. They were expressing either the calcium reporter GCaMP6s (fish 1-4, 6 and 8) or GCaMP6f (fish 5 and 7) under the control of the nearly pan-neuronal promoter elavl3 expressed in the nucleus Tg(elavl3:H2B-GCaMP6). Both lines were provided by Misha Ahrens and published by Vladimirov et al. (2014) (H2B-GCaMP6s) and Quirin et al. (2016) (H2B-GCaMP6f). Experiments were approved by Le Comité d'Éthique pour l'Experimentation Animale Charles Darwin C2EA-05 (02601.01).

\subsection{METHOD DETAILS}

\subsubsection{Light-sheet microscopy of zebrafish larvae}

Spontaneous neural activity (i.e. in the absence of sensory stimulation) was recorded in larval zebrafish using light-sheet microscopy, which acquires brain-scale scans by imaging multiple $z$-planes sequentially (Panier et al. 2013, Wolf et al. 2017, Migault et al., 2018). Larvae were placed in 2\% low melting point agarose (Sigma-Aldrich), drawn tail-first into a glass capillary tube with $1 \mathrm{~mm}$ inner diameter via a piston and placed in chamber filled with E3 in the microscope. Recordings were of length $1514 \pm 238$ seconds (mean \pm standard deviation), with a brain volume imaging frequency of $3.9 \pm 0.8 \mathrm{~Hz}$.

The following imaging pre-processing steps were performed offline using MATLAB, in line with previously reported protocols (Panier et al., 2013; Migault et al., 2018). Automated cell segmentation was performed using a watershed algorithm (Panier et al. 2013; Migault et al., 2018) and fluorescence values of pixels belonging 
to the same neuron was averaged to obtain cell measurements. The fluorescence intensity values $F$ were normalized to $\Delta F / F=(F-\langle F\rangle) /\left(\langle F\rangle-F_{0}\right)$ where $\langle F\rangle$ is the baseline signal per neuron and $F_{0}$ is the overall background intensity (Migault et al., 2018). The $\Delta F / F$ activity of different imaging planes was subsequently temporally aligned using interpolation (because of the time delay between imaging planes) (Migault et al. 2018) and deconvolved to binarized spike traces using Blind Sparse Deconvolution (BSD) (Tubiana et al. 2020). BSD estimates the most likely binary spike trace by minimizing the $L_{2}$ norm of the difference between the estimated spike trace convolved with an exponential kernel and the ground-truth calcium data, using $L_{1}$ sparsity regularization and online hyperparameter optimization. Calcium kernel time constants used for deconvolution were inferred using BSD on the spontaneous activity of three different fish (approximately 5000 neurons per fish, recorded at $10 \mathrm{~Hz}$, previously reported by Migault et al. (2018)). For the GCaMP6s line, we used a rise time of $0.2 \mathrm{~s}$ and a decay time of $3.55 \mathrm{~s}$; for the GCaMP6f line, we used $0.15 \mathrm{~s}$ and $1.6 \mathrm{~s}$, respectively.

Brain activity was recorded of 15 animals in total. Of these recordings, 1 was discarded because of poor image quality and 6 were discarded because neurons were inactive (defined by less than 0.02 spikes/(neurons $\times$ time points)), hence leaving 8 data sets for further analysis. The recorded brains were then registered onto the ZBrain Atlas (Randlett et al. 2015) and the mapzebrain atlas (Kunst et al. 2019) for anatomical labeling of neurons (Migault et al. 2018). The ZBrain Atlas was used in figures 1-4 because of its detailed region descriptions (outlining 294 regions in total). However, we also registered our data to the mapzebrain atlas (72 regions in total) in order to compare our results with the structural connectivity matrix which was defined for this atlas only (Kunst et al. 2019). Only neurons that were registered to at least 1 ZBrain region were used for analysis (to filter imaging artefacts). This resulted in $40709 \pm 13854$ neurons per recording (mean \pm standard deviation, minimum $=23446$, maximum $=65517$ ).

\subsubsection{Maximum Entropy Principle}

Here we provide in brief the general derivation of the class of maximum entropy probabilistic models. Restricted Boltzmann Machines are an instance of this model, which is detailed in the following sections. The maximum entropy principle is used to create probabilistic models $P(x)$ (where $x$ denotes one data configuration sample) that replicate particular data statistics $f_{k}$, but are otherwise most random, and therefore least assumptive, by maximizing their entropy $H=-\sum_{x} P(x) \log (P(x))$ (Gardella et al. 2019). The goal of the model is to match its model statistics $\left\langle f_{k}\right\rangle_{\text {model }}=\sum_{x} P(x) f_{k}(x)$ to the empirical data statistics $\left\langle f_{k}\right\rangle_{\text {data }}=F_{k}$. This is done using Lagrange multipliers $\Lambda_{k}$ :

$$
\widetilde{H}=-\sum_{x} P(x) \log (P(x))-\sum_{k} \Lambda_{k}\left(\sum_{x} P(x) f_{k}(x)-F_{k}\right)
$$

which yields, when $\widetilde{H}$ is maximized with respect to $P(x)$, the Boltzmann distribution (see e.g. Bialek (2012) for a full derivation):

$$
P(x)=\frac{1}{Z} \exp \left(-\ln 2 \sum_{k} \Lambda_{k} f_{k}(x)\right)=\frac{1}{Z} \exp (-E(x))
$$

where $E(x)$ is defined as the resulting energy function. Importantly, the data dependency $\left(F_{k}\right)$ disappears when going from Equation 5 to Equation 6 . Hence, the maximum entropy principle only defines the shape of the distribution $P(x)$, but not its specific parameters $\Lambda_{k}$ (Bialek .2012). In the case of RBM, these are then optimized using maximum likelihood estimation, as detailed in the sections below.

\subsubsection{Motivation for choice of statistics}

The derivation above describes the general maximum entropy model for a set of statistics $\left\{f_{k}\right\}$. The objective of this study is to extract the assembly structure from neural data, therefore creating two layers: a visible (neural data) layer $\mathbf{v}=\left(v_{1}, v_{2}, \ldots, v_{N}\right)$ and a hidden (latent) layer $\mathbf{h}=\left(h_{1}, h_{2}, \ldots, h_{M}\right)$. The model should capture the mean activity of each neuron $\left\langle v_{i}\right\rangle$, their pairwise correlations $\left\langle v_{i} v_{j}\right\rangle$, the neuron-HU interactions $\left\langle v_{i} h_{\mu}\right\rangle$ and a function of $h_{\mu}$. The latter is determined by the potential $\mathcal{U}$, which we set to be a double Rectified Linear Unit 
(dReLU), as motivated in the following sections. Fitting all $N^{2}$ pairwise interactions $\left\langle v_{i} v_{j}\right\rangle$ is computationally infeasible, but under the cell assembly hypothesis we assume that this should not be necessary because collective neural behavior is expected to be explained by membership to similar assemblies via $\left\langle v_{i} h_{\mu}\right\rangle$, and can therefore be excluded. We later show that pairwise correlations are indeed optimized implicitly (Figure 2). All other statistics are included and therefore explicitly optimized, also see Equation 15

\subsubsection{Restricted Boltzmann Machines}

A Restricted Boltzmann Machine (RBM) is an undirected graphical model defined on a bipartite graph (Smolensky, 1986; Hinton, 2002, Hinton \& Salakhutdinov, 2006), see Figure 2A. RBMs are constituted by two layers of random variables, neurons $\mathbf{v}$ and Hidden Units (HUs) $\mathbf{h}$, which are coupled by a weight matrix W. There are no direct couplings between pairs of units within the same layer. Here, each visible unit $v_{i}$ corresponds to a single recorded neuron with binary (spike-deconvolved) activity $\left(v_{i}(t) \in\{0,1\}\right)$. Each Hidden Unit (HU) $h_{\mu}$ corresponds to the (weighted) activity of its neural assembly and is chosen to be real-valued. The joint probability distribution $P(\mathbf{v}, \mathbf{h})$ writes (Hinton \& Salakhutdinov, 2006; Tubiana \& Monasson, 2017):

$$
P(\mathbf{v}, \mathbf{h})=\frac{1}{Z} \exp (-E(\mathbf{v}, \mathbf{h}))=\frac{1}{Z} \exp \left(\sum_{i} g_{i} v_{i}-\sum_{\mu} \mathcal{U}_{\mu}\left(h_{\mu}\right)+\sum_{i, \mu} w_{i, \mu} v_{i} h_{\mu}\right)
$$

Where $E$ is the energy function and $Z=\sum_{\mathbf{v}} \int_{\mathbf{h}} d v d h \cdot \exp (-E(\mathbf{v}, \mathbf{h}))$ is the partition function. The weights $g_{i}$ and potentials $\mathcal{U}_{\mu}$ control the activity level of the visible units and the marginal distributions of the HUs respectively, and the weights $w_{i, \mu}$ couple the two layers.

\subsubsection{From data to features}

Given a visible layer configuration $\mathbf{v}$, a HU $h_{\mu}$ receives the input $I_{\mu}(\mathbf{v})=\sum_{i} w_{i \mu} v_{i} \equiv \mathbf{w}_{\mu}{ }^{T} \mathbf{v}$ and, owing to the bipartite architecture, the conditional distribution $P(\mathbf{h} \mid \mathbf{v})$ factorizes as:

$$
P(\mathbf{h} \mid \mathbf{v})=\prod_{\mu} P\left(h_{\mu} \mid \mathbf{v}\right)=\prod_{\mu} \exp \left(\mathcal{U}_{\mu}\left(h_{\mu}\right)+h_{\mu} I_{\mu}(\mathbf{v})-\Gamma_{\mu}(I)\right)
$$

Where $\Gamma_{\mu}(I)=\log \left(\int_{h} d h \cdot \exp \left(-\mathcal{U}_{\mu}(h)+h I\right)\right)$ is the cumulant generating function associated to the potential $\mathcal{U}_{\mu}$ that normalizes Equation 8 (Tubiana et al. 2019b). The average activity of $\mathrm{HU} h_{\mu}$ associated to a visible configuration $\mathbf{v}$ is given by a linear-nonlinear transformation (as defined by the properties of the cumulant generating function):

$$
\left\langle h_{\mu} \mid \mathbf{v}\right\rangle=\frac{\partial \Gamma_{\mu}\left(I_{\mu}(\mathbf{v})\right)}{\partial I}=\Gamma_{\mu}^{\prime}\left(\mathbf{w}_{\mu}^{T} \mathbf{v}\right)
$$

Throughout the manuscript, we use this definition to compute HU activity $h_{\mu}(t)=\left\langle h_{\mu} \mid \mathbf{v}(t)\right\rangle$ (e.g. in Figure 4 .

\subsubsection{From features to data}

Conversely, given a hidden layer configuration $\mathbf{h}$, a visible unit $v_{i}$ receives the input $I_{i}(\mathbf{h})=\sum_{\mu} w_{i, \mu} h_{\mu} \equiv \mathbf{w}_{\mathbf{i}}^{T} \mathbf{h}$ and the conditional distribution factorizes as:

$$
P(\mathbf{v} \mid \mathbf{h})=\prod_{i} P\left(v_{i} \mid \mathbf{h}\right) \propto \prod_{i} \exp \left(\left(g_{i}+I_{i}(\mathbf{h})\right) v_{i}\right)
$$

and the average sampled $v_{i}$ activity is given by:

$$
\left\langle v_{i} \mid \mathbf{h}\right\rangle=\sigma\left(\mathbf{w}_{\mathbf{i}}^{T} \mathbf{h}+g_{i}\right)
$$

Where $\sigma(x)=1 /\left(1+e^{-x}\right)$ is the logistic function. Hence, a sampled visible layer configuration $\mathbf{v}$ is obtained by a weighted combination of the HU activity followed by Bernoulli sampling. RBMs are generative models, in the sense that they can generate new, artificial data using Equations 8 and 10 . Figure $2 \mathrm{~B}$ illustrates this Markov Chain Monte Carlo (MCMC) process, by recursively sampling from $P(\mathbf{h} \mid \mathbf{v})$ and $P(\mathbf{v} \mid \mathbf{h})$, which converges at equilibrium to $P(\mathbf{v}, \mathbf{h})$. 


\subsubsection{Marginal distributions}

The marginal distribution $P(\mathbf{v})$ has a closed-form expression because of the factorized conditional distribution of Equation 9 (Tubiana et al. 2019a b):

$$
P(\mathbf{v})=\int \prod_{\mu=1}^{M} d h_{\mu} \cdot P(\mathbf{v}, \mathbf{h})=\frac{1}{Z} \exp \left(\sum_{i=1}^{N} g_{i} v_{i}+\sum_{\mu=1}^{M} \Gamma_{\mu}\left(I_{\mu}(\mathbf{v})\right)\right)
$$

For a quadratic potential $\mathcal{U}_{\mu}(h)=\frac{\gamma_{\mu} h_{\mu}^{2}}{2}+\theta_{\mu} h_{\mu}$, the cumulant generating function would also be quadratic and $P(\mathbf{v})$ would reduce to a Hopfield model, i.e. a pairwise model with an interaction matrix $J_{i j}=\sum_{\mu} \frac{w_{i \mu} w_{j \mu}}{\gamma_{\mu}}$ (Tubiana

et al., 2019a). Otherwise, $\Gamma_{\mu}$ is not quadratic, yielding high-order effective interaction terms between visible units and allowing RBMs to express more complex distributions. Importantly, the number of parameters remains limited, controlled by $M$ and does not scale as $N^{2}$ (unlike pairwise models).

The marginal distribution $P(\mathbf{h})$ can be similarly expressed:

$$
P(\mathbf{h})=\frac{1}{Z} \exp \left(\sum_{\mu=1}^{M} \mathcal{U}_{\mu}\left(h_{\mu}\right)+\sum_{i=1}^{N} \log \left(1+\exp \left(g_{i}+I_{i}(\mathbf{h})\right)\right)\right)
$$

\subsubsection{Choice of HU potential}

The choice of HU potential determines three related properties: the HU conditional distribution $P(\mathbf{h} \mid \mathbf{v})$, the transfer function of the HUs and the parametric form of the marginal distribution $P(\mathbf{v})$. Hereafter we use the double-Rectified Linear Unit (dReLU) potential:

$$
\mathcal{U}_{\mu}(h)=\frac{1}{2} \gamma_{\mu,+} h_{+}^{2}+\frac{1}{2} \gamma_{\mu,-} h_{-}^{2}+\theta_{\mu,+} h_{+}+\theta_{\mu,-} h_{-}, \text {where } h_{+}=\max (h, 0), h_{-}=\min (h, 0)
$$

$\Longleftrightarrow \gamma_{\mu,-}, \theta_{\mu,-}$ and $w_{i \mu} \Longleftrightarrow-w_{i \mu} \forall i, \mu$ (leading to $h_{\mu} \Longleftrightarrow-h_{\mu}$ ). For visual clarity, we perform this sign swap transformation after training on all HUs with predominantly negative weights (defined by $\sum_{i} w_{i, \mu}<0$ ). Subsequently all HUs are positively activated if the group of neurons to which it connects is strongly active.

\subsubsection{RBM training}

The RBM is trained by maximizing the average log-likelihood of the empirical data configurations $\mathcal{L}=\langle\log P(\mathbf{v})\rangle_{\text {data }}$, using stochastic gradient descent methods. The gradient update steps are derived by calculating the derivative of $\mathcal{L}$, using Equation 12, with respect to the model parameters (Tubiana et al., 2019a):

$$
\begin{aligned}
\frac{\partial \mathcal{L}}{\partial g_{i}} & =\left\langle v_{i}\right\rangle_{\text {data }}-\left\langle v_{i}\right\rangle_{\text {model }} \\
\frac{\partial \mathcal{L}}{\partial w_{i \mu}} & =\left\langle v_{i} h_{\mu}\right\rangle_{\text {data }}-\left\langle v_{i} h_{\mu}\right\rangle_{\text {model }} \\
\frac{\partial \mathcal{L}}{\partial \theta_{\mu, \pm}} & =-\left\langle h_{\mu}^{ \pm}\right\rangle_{\text {data }}+\left\langle h_{\mu}^{ \pm}\right\rangle_{\text {model }} \\
\frac{\partial \mathcal{L}}{\partial \gamma_{\mu, \pm}} & =-\frac{1}{2}\left\langle h_{\mu}^{ \pm 2}\right\rangle_{\text {data }}+\frac{1}{2}\left\langle h_{\mu}^{ \pm 2}\right\rangle_{\text {model }}
\end{aligned}
$$


Each gradient of $\mathcal{L}$ is thus the difference between a data statistic $\left\langle f_{k}\right\rangle_{\text {data }}$ and a model statistic $\left\langle f_{k}\right\rangle_{\text {model }}$. Hence the model learns to match these statistics to the training data. Importantly, model statistics $\left\langle f_{k}\right\rangle_{\text {model }}$ cannot be evaluated exactly due to the exponentially large number of data configurations (e.g., $2^{N}$ visible configurations). Therefore they are approximated by computing the statistics of model-generated data using the MCMC sampling scheme defined with Equations 8 and 10 . MCMC sampling of a Boltzmann distribution in such highdimensional space is in general very challenging owing to the exponentially long time to reach equilibrium. We use the persistent contrastive divergence approximation (Tieleman, 2008) and discuss its validity below.

\subsubsection{Compositional Restricted Boltzmann Machine}

In the previous sections we have described the general properties of RBMs. We now motivate the specific RBM model choices that we have implemented, such as the dReLU potential and sparsity regularization, by discussing their impact on the properties of RBM-generated data.

Directed graphical models, e.g., PCA, ICA, sparse dictionaries or variational autoencoders, prescribe a priori statistical constraints for their data representations, such as orthogonality/independence or specific marginal distributions such as Gaussian/sparse distributions. In contrast, the statistical properties of the representation of the data learned by RBMs are unknown a priori by construction (because of the maximum entropy principle). Instead, they emerge from the structure of the weight matrix, the potentials and the recursive back-and-forth sampling procedure described above. We have therefore previously studied the properties of typical samples of RBM with random weights as a function of the visible and hidden unit potentials and properties of the weight matrix using statistical mechanics tools (Tubiana \& Monasson, 2017; Tubiana et al., 2019a). We have identified the three following typical behaviors, or phases.

In the ferromagnetic phase, a typical sample from $P(\mathbf{v}, \mathbf{h})$ has a single strongly activated $\mathrm{HU}(m(t) \sim 1$, where $m(t)$ is the number of activated HUs at time $t$ ), whereas the others are not or merely weakly activated. The corresponding active visible units $v_{i}$ are defined by the weight vector $\mathbf{w}_{\mu^{\star}}$ associated to the active HU $h_{\mu^{\star}}$ (see Equation 10 .

In the spin-glass phase, a typical sample does not have any relatively strongly activated HUs, but instead many moderately activated ones $(m(t) \sim M)$. They interfere in a complex fashion to produce different visible ration.

Finally, in the compositional phase, a typical sample from $P(\mathbf{v}, \mathbf{h})$ has a small number of strongly activated HUs $(1 \ll m(t) \ll M)$ whereas the others are weak or silent. Their weights are linearly combined through Equation 10 to produce the corresponding visible layer configuration. The compositional phase is desirable because, firstly, there exists a simple link between the weight matrix and typical data configurations (they are obtained by combining a few weights), which facilitates interpretation of biological systems (Tubiana et al. 2019b). Secondly, the corresponding neural activity distribution is rich, as different choices of HU subsets yield a combinatorial diversity of visible layer configurations. Moreover, the modular nature of the compositional phase facilitates the assembly organization of neural dynamics, as motivated in the Introduction.

A set of sufficient conditions for the emergence of the compositional phase are (Tubiana \& Monasson 2017):

1. The HUs are unbounded and real-valued with a ReLU-like transfer function.

2. The weight matrix $\mathbf{W}$ is sparse.

3. The columns $\mathbf{w}_{\mu}$ of the weight matrix have similar norm. (If a weight column associated to one $\mathrm{HU}$ is much larger than the others, visible configurations are solely aligned to it according to Equation 10 .)

The first condition is satisfied by the dReLU potential (but not by quadratic potentials or binary-valued HUs). The second condition is enforced in practice by adding a $L_{1}$ sparse penalty term $\lambda \cdot \sum_{i \mu}\left|w_{i \mu}\right|$ to the log-

likelihood cost function. In our experiments, the optimal sparsity parameter $\lambda$ was determined to be $\lambda=0.02$ 
by cross-validation (Figure S1). The final condition is achieved by enforcing that $\operatorname{Var}\left(h_{\mu}\right)=1$ and $\left\langle h_{\mu}\right\rangle \sim 0 \forall \mu$. This is done by an appropriate reparameterization of the HU potential of Equation 14 and a batch-norm-like procedure, described in detail in (Tubiana, 2018). This normalization promotes homogeneity among HU importance, preventing some units from being disconnected or others from dominating. In addition, ensuring that $h_{\mu}=O(1)$ irrespective of the visible layer size (as opposed to e.g. $\frac{1}{2}\left(\gamma_{+}+\gamma_{-}\right)=1$ which yields $h_{\mu} \sim \sqrt{N}$ ) avoids the problem of ill-conditioned Hessians that was previously described by Hinton (2012).

To emphasise the departure from the classic RBM formulation in this study, we name our model compositional RBM (cRBM).

\subsubsection{Hyperparameter settings}

The following hyperparameters were used in the experiments of this manuscript:

- Number of hidden unit $M: 200$. The value was determined by cross-validation (Figure S1) on one data set (example fish \#3). This value was then used for all other data sets, except for 3 data sets which used $M=100$ because their $N \approx \frac{1}{2} N_{\# 3}$.

- Sparse regularization penalty $\lambda$ : 0.02 (determined by cross-validation).

- Batch size: 100.

- Number of Monte Carlo chains: 100.

- Number of gradient updates: $2 \cdot 10^{5}$

- Number of Monte Carlo steps between each gradient update: 15 .

- Initial learning rate $\eta$ : between $5 \cdot 10^{-4}$ and $5 \cdot 10^{-3}$. We used $5 \cdot 10^{-3}$ by default and if weight divergence was observed, the learning was reinitialized with a reduced learning rate. This occurred notably for high- $M$ and low- $\lambda$ models during the cross-validation procedure of Figure $\mathbf{S 1}$

- Learning rate annealing scheme: the learning rate geometrically decayed during training, starting after $25 \%$ of the gradient update steps, from its initial value $\eta$ to a final value of $10^{-5}$. 
- Number of training data samples: $70 \%$ of frames of each recording (= 4086 training data samples on average), see section 7.4.1.1 for details.

\subsubsection{Functional Connectivity Inference}

\subsubsection{Effective connectivity matrix}

In this section we present a derivation of the effective coupling matrix between neurons from the marginal distribution $P(\mathbf{v})$ using cRBMs. We first define the local coupling $J_{i j}$ between two neurons $v_{i}$ and $v_{j}$ for a generic probability distribution $P\left(v_{1}, v_{2}, . ., v_{N}\right)$, given a data configuration $\mathbf{v}$ :

$$
\begin{aligned}
J_{i j}(\mathbf{v}) & =\log \left(\frac{P\left(v_{i}=1 \mid v_{1}, \ldots, v_{i-1}, v_{i+1}, v_{j}=1, \ldots, v_{N}\right)}{P\left(v_{i}=1 \mid v_{1}, \ldots, v_{i-1}, v_{i+1}, v_{j}=0, \ldots, v_{N}\right)}\right) \\
& -\log \left(\frac{P\left(v_{i}=0 \mid v_{1}, \ldots, v_{i-1}, v_{i+1}, v_{j}=1, \ldots, v_{N}\right)}{P\left(v_{i}=0 \mid v_{1}, \ldots, v_{i-1}, v_{i+1}, v_{j}=0, \ldots, v_{N}\right)}\right)
\end{aligned}
$$

In other words, $J_{i j}$ is defined as the impact of the state of neuron $j$ on neuron $i$ in the context of activity pattern $\mathbf{v}$. Hence, the effective connectivity matrix $\mathbf{J}$ mathematically defines the functional connections, related (anti-correlated) collective behavior of neurons $i, j$. This effective coupling value is symmetric (because of Bayes' rule): $J_{i j}(\mathbf{v})=J_{j i}(\mathbf{v})$. For context, note that $J_{i j}(\mathbf{v})$ is uniformly zero for an independent 
model of the form $P\left(v_{1}, . ., v_{N}\right)=\prod_{i} P_{i}\left(v_{i}\right)$, and that for a maximum entropy pairwise (Ising) model, with $P\left(v_{1}, . ., v_{N}\right)=\frac{1}{Z} \exp \left(\sum_{i} g_{i} v_{i}+\sum_{i<j} J_{i j}^{\text {ising }} v_{i} v_{j}\right)$, the $J_{i j}(\mathbf{v})$ matrix exactly identifies with the coupling matrix $J_{i j}^{\text {ising }}$, and does not depend on the data configuration $\mathbf{v}\left(\right.$ so $J_{i j}(\mathbf{v})=J_{i j}$ ).

However, in general, and for RBMs in particular, $J_{i j}(\mathbf{v})$ depends on the data set $\mathbf{v}$, and an overall coupling matrix can be derived by taking its average over all data configurations:

$$
J_{i j}=\left\langle J_{i j}(\mathbf{v})\right\rangle_{\text {data }}
$$

Although Equation 17 has a closed-form solution for RBMs (by inserting Equation 12, a naive evaluation requires $O\left(N^{3} M T\right)$ operations where $T$ is the number of data samples. However, a fast and intuitive approximation can be derived by performing a second order Taylor expansion of $\Gamma_{\mu}\left(I_{\mu}\right)$ :

$$
J_{i j}=\sum_{\mu=1}^{M} w_{i \mu} w_{j \mu}\left\langle\Gamma_{\mu}^{\prime \prime}(\mathbf{v})\right\rangle_{\text {data }}=\sum_{\mu=1}^{M} w_{i \mu} w_{j \mu}\left\langle\operatorname{Var}\left(h_{\mu} \mid \mathbf{v}\right)\right\rangle_{\text {data }}
$$

Equation 18 is exact for quadratic potential and in general justified as the contribution of neurons $i, j$ is small compared to the scale of variation of $\Gamma_{\mu}, O(\sqrt{p N})$ where $p$ is the fraction of non-zero couplings. In conclusion, we have mathematically derived the effective coupling between any two neurons $i$ and $j$. Intuitively, two neurons $i, j$ are effectively connected if they are connected to the same hidden unit $\mu$ (Equation 18.).

\subsubsection{From inter-neuron to inter-region connectivity}

In the above section we have derived the inter-neuronal connectivity matrix $\mathbf{J}$. This matrix is then aggregated to an inter-regional connectivity matrix $\mathbf{J}^{\mathcal{R}}$ by taking the $L_{1}$ norm of the corresponding $\mathbf{J}$ matrix block (i.e. $J_{k m}^{\mathcal{R}}=\sum_{i \in R_{k}, j \in R_{m}}\left|J_{i j}\right| /\left(N_{R_{k}} \cdot N_{R_{m}}\right)$, where $R_{k}$ is the set of neurons in region $k$ ).

Next, to derive the average connectivity matrix across multiple recordings, we used a weighted average of the individual recordings, with a region-pair specific weight equal to the length of the recording multiplied by the sum of the number of neurons in both regions (also see Section 7.4.7). Compared to a naive average, this weighted average accounts for the variable number of neurons per region between recordings.

\subsubsection{Training cRBM models for connectivity estimates}

Constructing the functional connectivity matrix of a cRBM does not require test data, but just the estimated weight matrix $\mathbf{W}$ (as explained above). Therefore we trained new cRBMs using the entire recordings (100\% of data) to fully use the information available. cRBM training is stochastic, and to mitigate the possible variability that could arise we trained 5 cRBMs for each recording. Then, to assess convergence, we selected all cRBMs with $0.01<\operatorname{std}(\mathbf{w})<0.1$, where std denotes standard deviation, for further functional connectivity analysis (yielding 23 cRBMs for 8 data sets in total). Connectivity estimates of multiple cRBM models per data sets were averaged.

\subsubsection{Connectivity inference baselines}

We considered four additional connectivity inference baseline methods:

- The covariance matrix.

- The Pearson correlation matrix.

- The sparse inverse covariance matrix inferred by graphical LASSO (Friedman et al. 2008) (as implemented in scikit-learn with default settings (Pedregosa et al. 2011)). Graphical LASSO is an efficient method for inference of large scale connectivity. Unfortunately, the implementation available failed to converge in reasonable time due to the high dimensionality of the data. 
- The Ising model with pseudo-likelihood maximization (PLM) inference (Ravikumar et al., 2010).

Results obtained with the covariance and correlation matrices are presented in Figure S6. The connectivity matrices obtained by the PLM Ising model (not shown) correctly identified the diagonal entries of the regionregion matrix, but not the off-diagonal coefficients and had a weaker correlation with the structural connectivity matrix than the covariance and correlation matrices ( $r_{S}=0.06$ using 4 fish).

\subsection{QUANTIFICATION AND STATISTICAL ANALYSIS}

\subsubsection{Optimizing the free parameters of cRBM}

We set the free parameters $\lambda$ (sparsity regularization parameter) and $M$ (number of HUs) by cross-validating a large range of $(\lambda, M)$ values for one data set (fish \#3). This was done by training cRBMs on $70 \%$ of the data, and evaluating model performance on the remaining test data, as detailed below. The resulting optimal values could then be used for all data sets (where $M$ was scaled with the number of neurons $N$ ).

\subsubsection{Train / test data split}

We split up one recording (fish \#3) into training data (70\% of recording) and withheld test data (30\% of recording) for the free parameter $(\lambda, M)$ optimization procedure. This enabled us to assess whether the cRBMs learned to model the data statistics (as described in the main text, Figures 2] and S1), while ensuring that the cRBMs had not overfit to the specific training data configurations. Importantly, this assumes that the test data comes from the same statistical distribution as the training data (while consisting of different data configurations). To ensure this, we split up the recording of example fish \#3 (used for parameter optimization) in training and test splits as follows (before training the cRBMs): We divided the recording of length $T$ in 10 chronological segments of equal length (so that segment 1 has time points $\left\{t \in\left[1, \frac{T}{10}\right)\right\}$ et cetera), with the rationale that by maintaining

$\left(\begin{array}{c}10 \\ 3\end{array}\right)=120$ possible training/test splits of the neural data. We then evaluated the statistical similarity between the training and test split of each combination, by assessing the mean neural activity $\left\langle v_{i}\right\rangle$ and pairwise neural correlations $\left\langle v_{i} v_{j}\right\rangle-\left\langle v_{i}\right\rangle\left\langle v_{j}\right\rangle$ statistics. We quantified the similarity between training and test statistics by calculating the Root Mean Square Error $\left(\operatorname{RMSE}\left(\mathbf{x}_{1}, \mathbf{x}_{2}\right)=\sqrt{\frac{1}{N_{x}} \sum_{n=1}^{N_{x}}\left(x_{1}(n)-x_{2}(n)\right)^{2}}\right)$. The most similar split is defined by the lowest RMSE, but to show that $\mathrm{cRBM}$ are not dependent on picking the best possible split, but rather on avoiding the bad splits, we then chose to use the split with the $10^{\text {th }}$-percentile ranking RMSE. We hope that this aids future studies, where a potentially high number of possible splits prevents researchers from evaluating all possible splits, but a good split may nevertheless be found efficiently.

\subsubsection{Assessment of data statistics}

Firstly, we evaluated 3 statistics that cRBMs are trained to optimize: the mean activity of neurons $\left\langle v_{i}\right\rangle$, the mean activity of HUs $\left\langle h_{\mu}\right\rangle$ and their pairwise interactions $\left\langle v_{i} h_{\mu}\right\rangle$. Additionally, second order statistics of pairwise neuron-neuron interactions $\left\langle v_{i} v_{j}\right\rangle$, HU-HU interactions $\left\langle h_{\mu} h_{v}\right\rangle$ and the reconstruction quality were evaluated, which the cRBM was not constrained to fit. Data statistics were calculated on withheld test data (30\% of recording). Model statistics cannot be calculated exactly, because that would require one to sample all possible states $P(\mathbf{v}, \mathbf{h})$, and were therefore approximated by evaluating cRBM-generated data. Here, 300 Monte Carlo chains were each initiated on random training data configurations and 50 configurations were sampled consecutively for each chain, with 20 sampling steps between saved configurations, after a burn-in period of 100 effective sampling configurations.

The $\left\langle v_{i} h_{\mu}\right\rangle$ statistic (Figure 2 $\mathrm{C}$ ) was corrected for the sparsity regularization, by adding the sparsity regularization parameter $\lambda$ to $\left\langle v_{i} h_{\mu}\right\rangle:\left\langle v_{i} h_{\mu}\right\rangle_{\text {model }}=\left\langle v_{i} h_{\mu}\right\rangle_{\text {model-generated data }}+\lambda \cdot \operatorname{sign}\left(w_{i, \mu}\right)$. Furthermore, $\left(v_{i}, h_{\mu}\right)$ pairs with exactly $w_{i, \mu}=0$ were excluded from analysis (5\% of total for optimal cRBM in Figure $2 \mathrm{C}$ ).

The pairwise neuron-neuron and HU-HU statistics $\left(\left\langle v_{i} v_{j}\right\rangle,\left\langle h_{\mu} h_{v}\right\rangle\right)$ were corrected for their (trivially) expected correlation due to their mean activities (by subtraction of $\left\langle v_{i}\right\rangle\left\langle v_{j}\right\rangle$ and $\left\langle h_{\mu}\right\rangle\left\langle h_{\nu}\right\rangle$ respectively), so that only 
true correlations were assessed.

Goodness of fit was quantified by computing the normalized Root Mean Square Error (nRMSE) for each statistic (shown in Figure S1). The RMSE between two vectors $\mathbf{x}_{1}, \mathbf{x}_{2}$ of length $N_{x}$ is defined as RMSE $=$ $715 \sqrt{\frac{1}{N_{x}} \sum_{n=1}^{N_{x}}\left(x_{1}(n)-x_{2}(n)\right)^{2}}$. Ordinary RMSE was normalized so that different statistics could be compared, where 1 corresponds to $\mathrm{nRMSE}_{\text {shuffled, }}$, where both data and model statistics were randomly shuffled, and 0 corresponds to $\mathrm{nRMSE}_{\text {optimal }}$ which is the RMSE between the training data and test data (by nRMSE $=$ $1-\frac{\mathrm{RMSE}_{\text {ordinary }}-\mathrm{RMSE}_{\text {shuffled }}}{\mathrm{RMSE}_{\text {optimal }}-\mathrm{RMSE}_{\text {shuffled }}}$.

Additionally, we assessed the reconstruction quality of the test data. Here, the log-likelihood (LLH) between the test data $\mathbf{v}$ and its reconstruction $\mathbb{E}\left(\mathbf{v}_{\text {recon }}\right)=\mathbb{E}(\mathbf{v} \mid \mathbb{E}(\mathbf{h} \mid \mathbf{v})) \in[0,1]$ was computed. Because $v_{i} \in\{0,1\}$, the LLH is defined as

$$
\operatorname{LLH}\left(v_{i}, \mathbb{E}\left(\mathbf{v}_{\text {recon }}\right)\right)=\frac{1}{T} \sum_{t=1}^{T} \log \left(\mathbb{E}\left(\mathbf{v}_{\text {recon }}\right)(t) * v_{i}(t)+\left(1-\mathbb{E}\left(\mathbf{v}_{\text {recon }}\right)(t)\right) *\left(1-v_{i}(t)\right)\right)
$$

The resulting LLH was normalized (nLLH) such that 0 corresponds to an independent model (i.e. fitting neural activity with $\mathbb{E}\left(\mathbf{v}_{\text {recon }}\right)(t)=\left\langle v_{i}\right\rangle \forall t$ ) and 1 to optimal performance (which is $\mathrm{LLH}_{\text {optimal }}=0$ ), by nLLH $=$ $\frac{\mathrm{LLH}_{\text {ordinary }}-\mathrm{LLH}_{\text {independent }}}{-\mathrm{LLH}_{\text {independent }}}$.

\subsubsection{Generalized Linear Model}

We used logistic regression, a Generalized Linear Model (GLM), to quantify the reconstruction quality of a connected model (i.e. with neuron-to-neuron connections, see Figure S2A). Logistic regression makes a probabilistic binary prediction (Bishop. 2006), hence allowing direct comparison to the probabilistic estimates of neural activity by the cRBM. In logistic regression, for a neuron $v_{i}(t)$ at time $t$, the activity of all other neurons $\mathbf{v}_{-i}(t)$ at time $t$ was used to predict $\hat{v}_{i}(t)=P\left(v_{i}(t)=1\right)=\frac{1}{1+\exp \left(-\mathbf{w}_{i} \cdot \mathbf{v}_{-}(t)\right)}$ where $\mathbf{w}_{i}$ is the estimated weight vector. This was implemented with scikit-learn (Pedregosa et al. 2011$)$, using $L_{2}$ sparsity regularization. The sparsity parameter $\lambda_{\mathrm{GLM}}$ was optimized to $\lambda_{\mathrm{GLM}}=1000$ using cross-validation (Figure $\mathrm{S} 2 \mathrm{~B}$ ). This is a computationally intensive model to compute because of the large number of regressor neurons $N-1$. Therefore we performed the cross-validation of Figure S2B on $2 \%$ of all neurons (=1050 neurons) and computed the final distribution of Figure $2 \mathrm{H}$ on $10 \%$ of all neurons (=5252 neurons). GLMs were trained on the same train data as cRBMs, and evaluated on the same withheld test data as cRBMs (as described above).

\subsubsection{Regional occupancy}

We determined the anatomical region labels of each neuron by registering our recordings to the ZBrain Atlas (as described previously). This yields a matrix $L$ of size $N_{\mathrm{ZBA}} \times N$, which elements are $l_{r, i}=1$ if neuron $i$ is embedded in region $r$ and 0 if it is not. A cRBM neural assembly of $\mathrm{HU} \mu$ is defined by its weight vector $\mathbf{w}_{\mu}$ (of size $N$ ). Because cRBMs converge to sparse solutions, most of the weight elements will be very close to 0 . To determine which anatomical regions are occupied by the assembly neurons with significantly nonzero weights, we computed the dot product between the weight vector $\mathbf{w}_{\mu}$ and matrix $L$, leading to a weighted region label vector (of size $N_{\text {ZBA }}$ ) for each HU. The matrix of all $M$ weighted region label vectors is shown in Figure S4A for cRBM and Figure S4B for PCA.

The effective number of anatomical regions that one cRBM/PCA assembly is embedded in was then calculated using the Participation Ratio (PR) of each HU/Principal Axis. PRs are used to estimate the effective number of nonzero elements in a vector, without using a threshold (Tubiana \& Monasson, 2017). The PR of a vector $\mathbf{x}=\left(x_{1}, . ., x_{n}\right)$ is defined by:

$$
\operatorname{PR}(\mathbf{x})=\frac{\left(\sum_{i=1}^{n} x_{i}^{2}\right)^{2}}{\sum_{i=1}^{n} x_{i}^{4}}
$$


PR varies from $\frac{1}{n}$ when only 1 element of $\mathbf{x}$ is nonzero and $n$ when all elements are equal. We therefore estimated the effective number of regions by multiplying PR of the weighted region label vectors with the total number of regions $N_{\mathrm{ZBA}}$ in Figure $\mathrm{S} 4 \mathrm{C}$.

\subsubsection{Time constant calculation}

The dReLU potential $\mathcal{U}_{\mu}$ of equation 14 can learn to take a variety of shapes, including a double-well potential (Tubiana et al., 2019a). HUs generally converged to this shape, giving rise to bimodal HU activity distributions (Figure 4D). We determined the positions of the two peaks per HU using Gaussian Mixture Models fitted with two Gaussians. The bimodality transition point was then defined as the average between the two peaks (which was approximately 0 for most HUs). To calculate the time constant of state changes between the two activity modes, we subtracted the bimodality transition point from each HU activity $h_{\mu}$ individually. For clarity, all dynamic activity traces shown (e.g. Figure 4) are thus bimodality transition point subtracted. The time constant of an activity trace was then defined as the period of a (two-state) oscillation. A HU oscillation is defined as a consecutive negative and positive activity interval (because the bimodality now occurs at 0 ). A neuron oscillation is defined as a consecutive interspike-interval and spike-interval (which can last for multiple time steps, for example see Figure $1 \mathrm{~A}$, right panel).

\subsubsection{Sorting of HUs}

HUs were sorted by hierarchical clustering of the Pearson correlation matrix of their dynamic activity (Figure $4 \mathrm{~B}$ ). Hierarchical clustering was performed using the Ward variance minimization algorithm that defines the distance between clusters (Virtanen et al., 2020). This sorting of HUs (and thus assemblies) is maintained throughout the manuscript for the sake of consistency.

\subsubsection{Validating that the $\mathrm{CRBM}$ is in the compositional phase}

To validate that the cRBMs converged to the compositional phase (see section 7.3.4, compositional RBM formulation), we calculated the effective number of active HUs per data configuration (i.e. time step) $m(t)=$ $\operatorname{PR}\left(\mathbf{h}_{+}(t)\right) \cdot M$ where PR is the participation ratio (equation 20, $M$ the number of HUs and $h_{+}=h-h_{\text {inactive }}$, where $h_{\text {inactive }}$ is the inactive peak as calculated with the Gaussian Mixture Models (see section 7.4.3), because PR assumes that inactive elements are approximately zero (Tubiana et al. 2019a). A cRBM is said to be in the compositional phase if $1 \ll \operatorname{median}(m) \ll M$, which is true for all cRBMs (Figure S3).

\subsubsection{Extensions of the structural connectivity matrix}

The inter-region structural connectivity matrix was derived from the single cell zebrafish brain atlas (Kunst et al. 2019). We used the post-publication updated data set from (Kunst et al., 2019) (timestamp: 28 October 2019). The data set consists of $N=3098$ neurons, each characterized by the 3D coordinates of the soma center and of its neurites; there is no distinction between dendrites and axons. The brain is subdivided into $R=72$ regions and each neuron is duplicated by left/right hemisphere symmetry. We aim to estimate $c_{r, r^{\prime}}$, the average strength of the connection between two neurons belonging to regions $r, r^{\prime} \in[1, R]$. For each neuron $n \in[1, N]$, we determine, using region masks, the region $r(n)$ where its soma is located and the cumulative length of the intersection between all its neurites and each region $\ell_{n}(r)$. Under the assumptions that (i) the linear density of dendritic spines / axon presynaptic boutons is constant and (ii) the volumetric density of neurons is identical throughout regions, $L_{n}(r)$ is proportional to the volume $V_{r}$ of region $r$ times the average (bidirectional) connection strength between neuron $n$ and any neuron of region $r$. Aggregating over all neurons and symmetrizing, we obtain the following estimator for $c_{r, r^{\prime}}$ :

$$
c_{r, r^{\prime}}=\text { Symmetrized }\left\{\frac{\sum_{n=1}^{N} \delta_{r(n), r} \times \ell_{n}\left(r^{\prime}\right)}{V_{r^{\prime}} \times \sum_{n=1}^{N} \delta_{r(n), r}}\right\}
$$

where $\delta_{r(n), r}=1$ if neuron $n$ has its soma in region $r$ and 0 if not. Using the same notations, the formula previously used in (Kunst et al. 2019) is:

$$
c_{r, r^{\prime}}=\left\{\frac{\sum_{n=1}^{N} \ell_{n}(r)+\ell_{n}\left(r^{\prime}\right)}{N\left(V_{r^{\prime}}+V_{r}\right)}\right\}
$$


Equation 21 differs from Equation 22 in three aspects:

1. It discriminates between direct and indirect connections. Previously, a structural connection between region $r$ and region $r^{\prime}$ was established if a neuron had neurites with either tips or its soma within both regions. This may however result in indirect connections between $r$ and $r^{\prime}$, in cases where the neuron soma resides in another region $r^{\prime \prime}$. Here, we only account for direct connections, resulting in an overall slightly sparser connectivity matrix.

2. It is well-defined along the diagonal, i.e. for intra-region connections, whereas in Equation 22, each neurite would be counted as a self-connection.

3. The denominator corrects for non-uniform sampling of the traced neurons throughout regions. Note that this issue only arose in the post-publication data set as non-uniform sampling was used to fill missing entries of the matrix.

\subsubsection{Specimen averaging of connectivity matrices}

The number of neurons in a particular brain region can vary across recordings from different specimen.

Since the entries of the connectivity matrix are expected to be more accurate for well-sampled regions, we computed the weighted average of region-to-region connections $c_{r, r^{\prime}}$ as follows:

$$
\begin{array}{r}
\left\langle c_{r, r^{\prime}}\right\rangle=\frac{\sum_{\mathrm{Fish}} c_{r, r^{\prime}}^{F} \cdot w_{r, r^{\prime}}^{F}}{\sum_{\mathrm{Fish}} w_{r, r^{\prime}}^{F}} \\
w_{r, r^{\prime}}^{F}=T^{F} \frac{\left(N_{R_{r}}^{F}+N_{R_{r^{\prime}}}^{F}\right)}{2}
\end{array}
$$

Where $T^{F}$ is the recording length and $N_{R_{r}}^{F}$ is the number of neurons in region $r$ of fish $F$ that were recorded.

\subsubsection{Correlation analysis of connectivity matrices}

Pearson correlation was used to assess the similarity between cRBM functional connectivity matrices of different individual animals (Figure 5p. Spearman correlation was used to compare structural connectivity versus functional connectivity (Figure 6, because these two metrics do not necessarily scale linearly. All correlation analyses, and the Kilmogorov-Smirnov test of Figure S6C, performed on symmetric matrices excluded one off-diagonal triangle (of symmetrical values) to avoid duplicates. 


\subsection{KEY RESOURCES TABLE}

\begin{tabular}{|c|c|c|}
\hline REAGENT or RESOURCE & SOURCE & IDENTIFIER \\
\hline $\begin{array}{l}\text { Software and algorithms } \\
\text { cRBM algorithm } \\
\text { Fishualizer } \\
\text { Blind Sparse Deconvolution } \\
\text { ZBrain Atlas } \\
\text { mapzebrain atlas } \\
\text { MATLAB (data preprocessing) } \\
\text { Computational Morphometry Toolkit (CMTK) } \\
\text { Python }\end{array}$ & $\begin{array}{l}\text { This paper and } \\
\begin{array}{|l|}\text { Tubiana \& Monasson } \\
\text { Migault et al. }(2017) \\
\text { Tubiana et al. } \\
\text { Randlett et al. }(2020) \\
\text { Kunst et al. }(2015) \\
\text { MathWorks } \\
\text { NITRC } \\
\text { Python Software Foundation }\end{array}\end{array}$ & \begin{tabular}{|l|} 
github.com/jertubiana/PGM \\
\begin{tabular}{|l|} 
bitbucket.org/benglitz/fishualizer_public \\
github.com/jertubiana/BSD \\
engertlab.fas.harvard.edu/Z-Brain \\
fishatlas.neuro.mpg.de \\
mathworks.com/products/matlab.html \\
nitrc.org/projects/cmtk \\
python.org \\
\end{tabular} \\
\end{tabular} \\
\hline $\begin{array}{l}\text { Experimental Models: Organisms/Strains } \\
\operatorname{Tg}(\text { elav13:H2B-GCaMP6f }) \\
\text { Tg(elav13:H2B-GCaMP6s })\end{array}$ & \begin{tabular}{|l|l|} 
Vladimirov et al. & $(2014)$ \\
Vladimirov et al. & $(2014)$ \\
\end{tabular} & $\begin{array}{l}\text { N/A } \\
\text { N/A }\end{array}$ \\
\hline $\begin{array}{l}\text { Deposited Data } \\
\text { Functional neural activity recordings } \\
\text { Trained cRBM models }\end{array}$ & $\begin{array}{l}\text { This paper } \\
\text { This paper }\end{array}$ & $\begin{array}{l}\text { Will be made public upon publication } \\
\text { Will be made public upon publication }\end{array}$ \\
\hline
\end{tabular}




\section{Supplementary Information}

\begin{tabular}{ll|ll}
\hline Abbreviation & Full name & Abbreviation & Full name \\
\hline th & Thalamus left & s & Subpallium left \\
ts & Torus semicircularis left & mos2 & MO stripe 2 left \\
$\mathrm{t}$ & Tegmentum left & MOS2 & MO stripe 2 right \\
mos1 & MO stripe 1 left & vr & Vagal region left \\
TH & Thalamus right & OE & Olfactory epithelium right \\
TS & Torus semicircularis right & oe & Olfactory epithelium left \\
T & Tegmentum right & VR & Vagal region right \\
MOS1 & MO stripe 1 right & IO & Inferior olive right \\
MOS5 & MO stripe 5 right & nx & Vagus motor neurons left \\
mos4 & MO stripe 4 left & NX & Vagus motor neurons right \\
MOS4 & MO stripe 4 right & io & Inferior olive left \\
mos5 & MO stripe 5 left & tl & Torus longitudinalis left \\
mon & Medial octavolateral nucleus left & TL & Torus longitudinalis right \\
MON & Medial octavolateral nucleus right & TeO & Tectum right \\
Ce & Cerebellum right & teo & Tectum left \\
ce & Cerebellum left & OB & Olfactory bulb right \\
MOS3 & MO stripe 3 right & ob & Olfactory bulb left \\
mos3 & MO stripe 3 left & ha & Habenula left \\
Pr & Pretectum right & Ha & Habenula right \\
pr & Pretectum left & irf & Intermediate reticular formation left \\
PT & Posterior tuberculum right & prf & Posterior reticular formation left \\
pt & Posterior tuberculum left & r & Raphe nucleus left \\
$\mathrm{P}$ & Pallium right & R & Raphe nucleus right \\
p & Pallium left & iRF & Intermediate reticular formation right \\
$\mathrm{S}$ & Subpallium right & pRF & Posterior reticular formation right \\
\hline
\end{tabular}

Table S1: Table of abbreviations of mapzebrain atlas region names (used for interregional connectivity analyses). 
bioRxiv preprint doi: https://doi.org/10.1101/2021.11.09.467900; this version posted November 11,2021 . The copyright holder for this preprint (which was not certified by peer review) is the author/funder, who has granted bioRxiv a license to display the preprint in perpetuity. It is made available under aCC-BY-NC-ND 4.0 International license.
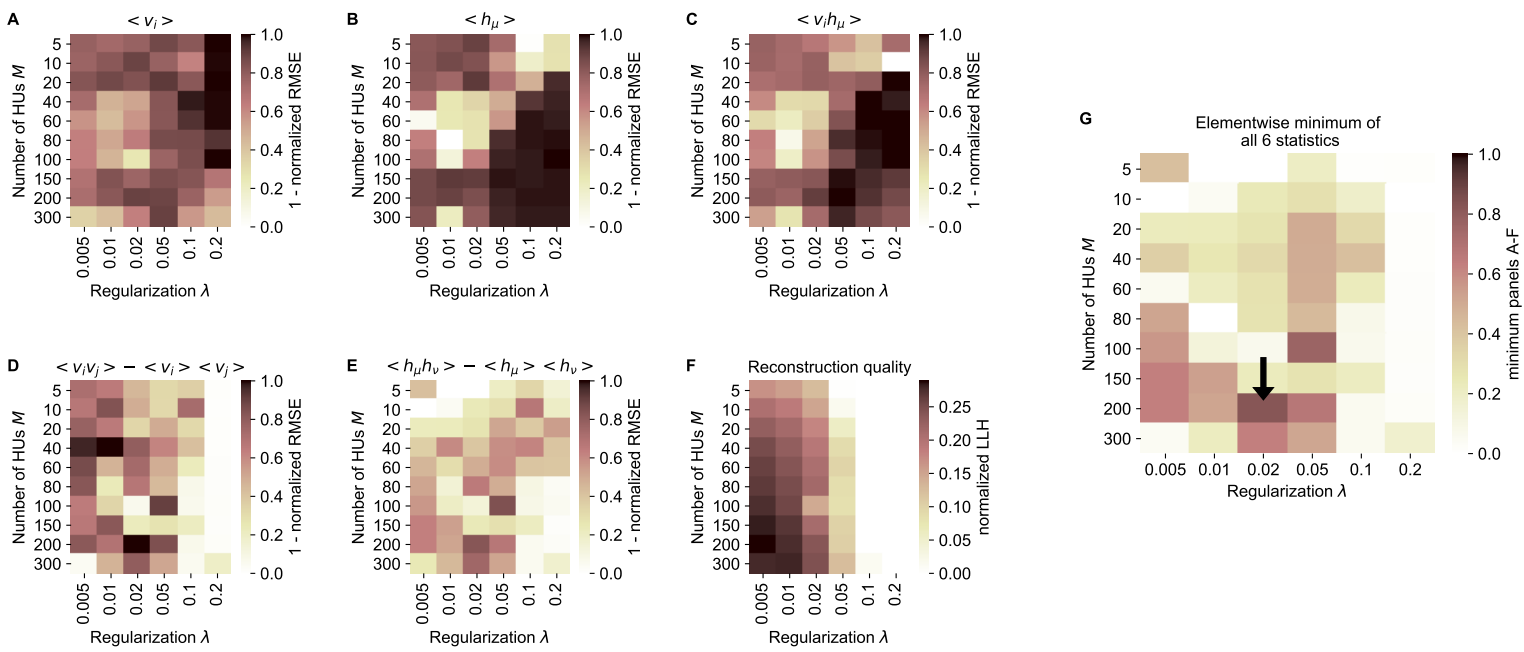

Figure S1: cRBM free parameter optimization by cross-validation

(A) Model performance, as quantified by the normalized RMSE (nRMSE, see STAR methods) of the mean neural activity $\left\langle v_{i}\right\rangle$, as a function of the sparsity regularization parameter $\lambda$ (x-axis) and the number of HUs $M$ (y-axis). cRBM models were evaluated after training converged on withheld testing data. $1-$ nRMSE values are shown to enable optimization by maximization in panel G. Values below 0 were set to 0 for panels A-F.

(B) Equivalent figure for the mean HU activity $\left\langle h_{\mu}\right\rangle$.

(C) Equivalent figure for average pairwise neuron-HU interactions $\left\langle v_{i} h_{\mu}\right\rangle$.

(D) Equivalent figure for average pairwise neuron-neuron interactions $\left\langle v_{i} v_{j}\right\rangle$.

(E) Equivalent figure for average pairwise HU-HU interactions $\left\langle h_{\mu} h_{v}\right\rangle$.

(F) Equivalent figure for the reconstruction quality of the low-dimensional cRBM bottleneck, quantified by the median normalized LLH.

(G) The cRBM free parameters were optimized by maximizing the element-wise minimum of panels A-F. Using the element-wise minimum to compare the six statistics ensures that the model performs performs well on all aspects. First, in order to compare panels A-E with panel F, the values of panel $\mathrm{F}$ were scaled to 1 by dividing all elements of panel $\mathrm{F}$ by its maximum value $(=0.29)$. Next, for each $(\lambda, M)$ combination the minimum value was determined from all 6 evaluation criteria. These are shown in this panel, with the black arrow indicating the resulting maximum of 0.83 at $(\lambda=0.02, M=200)$.
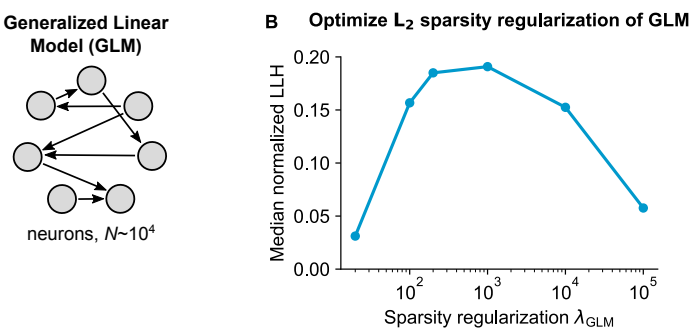

Figure S2: Generalized Linear Model (GLM) parameter optimization

(A) Schematic of the pairwise GLM model. Connections were estimated with logistic regression and $L_{2}$ sparsity regularization. (B) The sparsity regularization parameter $\lambda_{\mathrm{GLM}}$ was optimized using cross-validation of 6 parameter configurations, using 1000 randomly sampled neurons. 
bioRxiv preprint doi: https://doi.org/10.1101/2021.11.09.467900; this version posted November 11, 2021. The copyright holder for this preprint (which was not certified by peer review) is the author/funder, who has granted bioRxiv a license to display the preprint in perpetuity. It is made available under aCC-BY-NC-ND 4.0 International license.
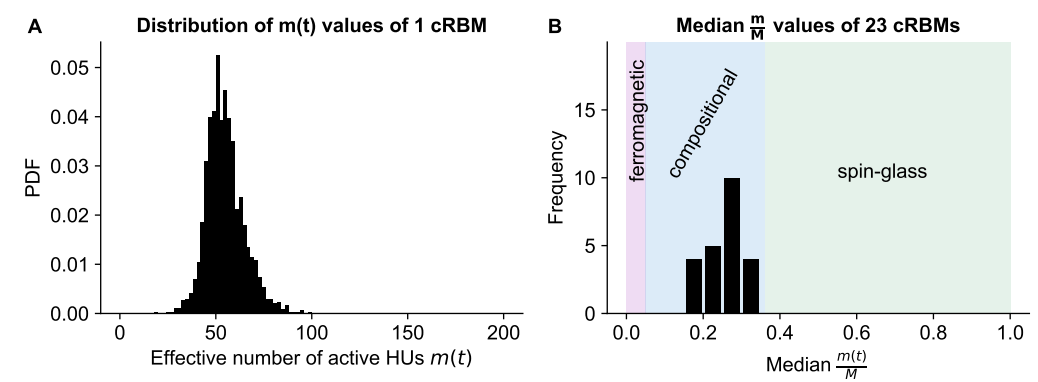

Figure S3: cRBMs are in the compositional phase after convergence

(A) Distribution of $m(t)$, the number of effective active HUs per time point $t$, calculated on the test data of example fish \#3. $m(t)$ is defined as $m(t)=\mathrm{PR}\left(\mathbf{h}_{+}(t)\right) \cdot M$, where PR is the Participation Ratio (Equation 20. Median $m(t)=0.27 \cdot M=54$.

(B) The distribution of median $\frac{m(t)}{M}$ values of the entire recordings of all cRBMs used for the connectivity analyses. The average $\frac{\text { median }(m)}{M}$ across all cRBMs is 0.26 . The compositional phase is characterized by $1 \ll m(t) \ll M$ which occurs for all cRBMs. The three phases are indicated. Here, the ferromagnetic phase upper bound was manually set at $5 \%$, and the spin-glass phase lower bound was determined by computing the PR of normally distributed activity (with mean and standard deviation of the test data of example fish \#3 of panel A).

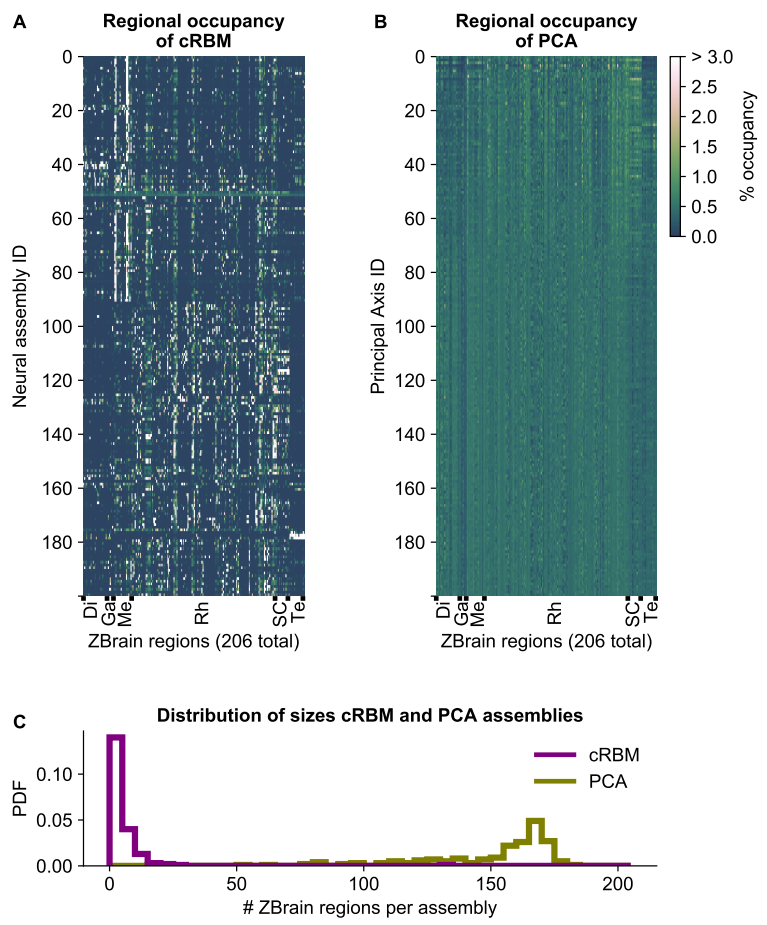

Figure S4: cRBM assemblies are sparse and spatially localized

(A) For each neural assembly (y-axis), the occupancy of ZBrain Atlas (Randlett et al. 2015) brain regions (x-axis, sorted alphabetically) was determined, by quantifying the number of anatomical regions that each $\mathrm{HU}$ connects to (i.e. the overlap between cRBM assemblies and anatomical regions, see STAR Methods). ZBrain atlas regions that were not imaged were excluded, yielding $N_{\mathrm{ZBA}}=206$ regions in total. Occupancy was defined as the dot product between the binary ZBrain-label matrix (size $N_{\mathrm{ZBA}} \times N$, where 1 indicates that a neuron is embedded in an anatomical region, and 0 vice versa) and the cRBM weight matrix (size $N \times M$ ), and was normalized to $100 \%$ for each assembly to account for different assembly sizes. Assemblies were sorted by their dynamics (Figure 4B, STAR methods). ZBrain Atlas regions are abbreviated by: Diencephalon (Di), Ganglia (Ga), Mesencephalon (Me), Rhombencephalon (Rh), Spinal Cord (SC) and Telencephalon (Te).

(B) Equivalent figure for Principal Axes of PCA (i.e. the PCA Eigenvectors). Principal Axes were sorted by Eigenvalue. Panels A and B share the same color scale (right).

(C) Distributions of the effective number of ZBrain Atlas regions per assembly (Principal Axis) of the occupancy metric of panel A (B) for cRBM (PCA) respectively. The effective number of regions was determined by calculating the Participation Ratio per assembly (Principal Axis), multiplied with the total number of regions $N_{\text {ZBA }}$ (see STAR methods). cRBM assemblies occupy a median of 3 regions (interquartile range: 2 to 6 regions), while PCA assemblies occupy a median of 160 regions (interquartile range: 140 to 168 regions). 
bioRxiv preprint doi: https://doi.org/10.1101/2021.11.09.467900; this version posted November 11, 2021. The copyright holder for this preprint (which was not certified by peer review) is the author/funder, who has granted bioRxiv a license to display the preprint in perpetuity. It is made available under aCC-BY-NC-ND 4.0 International license.
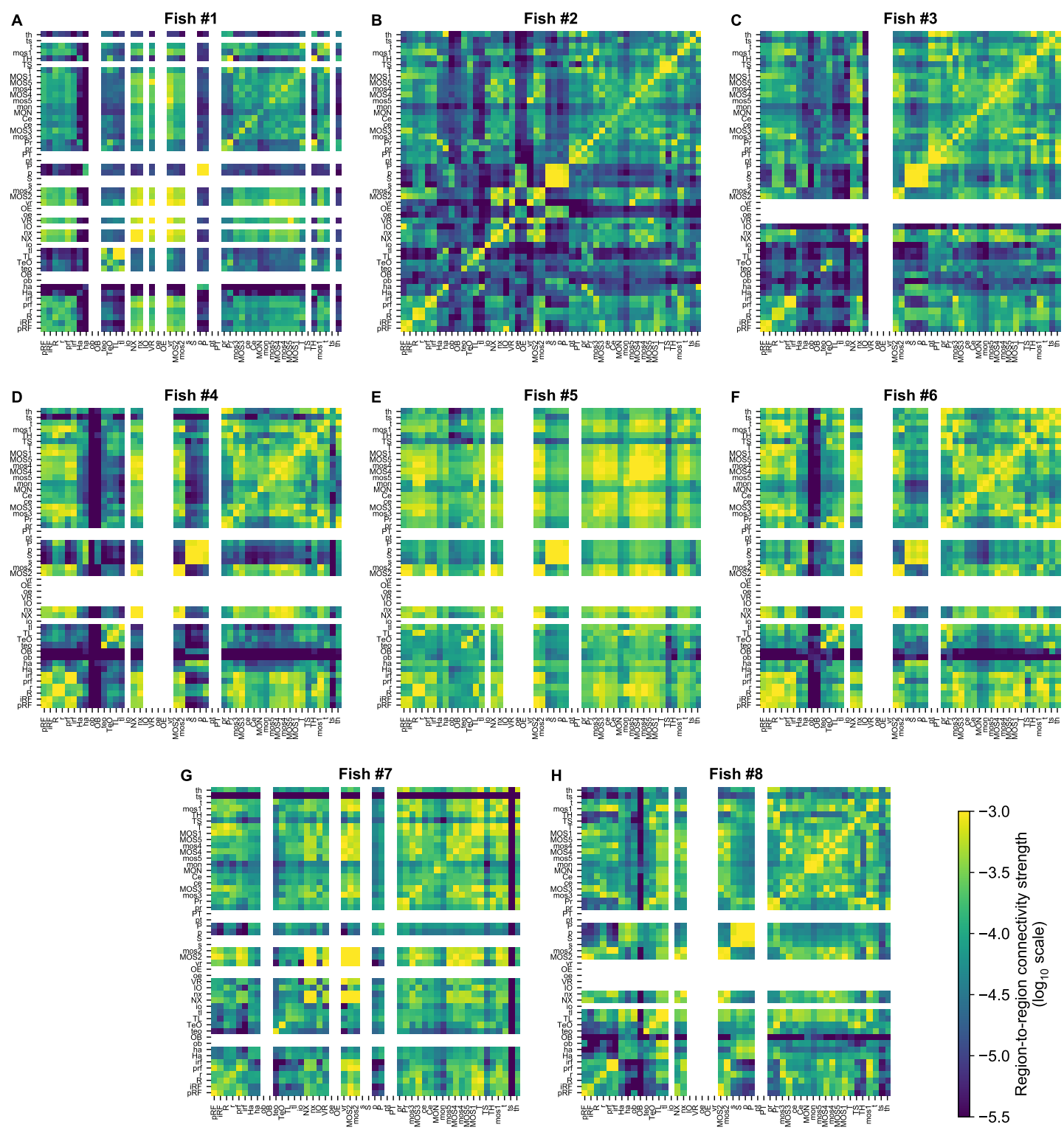

Figure S5: Functional connectivity matrices of all fish

8 panels (A-H) showing the individual cRBM functional connectivity matrices of all zebrafish recordings. Panels B, C and D correspond to Figures 5 A-C. All matrices share the same $\log _{10}$ color scale (bottom right). Regions that contained less than 5 neurons were left blank, for each fish individually. 
bioRxiv preprint doi: https://doi.org/10.1101/2021.11.09.467900; this version posted November 11,2021 . The copyright holder for this preprint (which was not certified by peer review) is the author/funder, who has granted bioRxiv a license to display the preprint in perpetuity. It is made available under aCC-BY-NC-ND 4.0 International license.

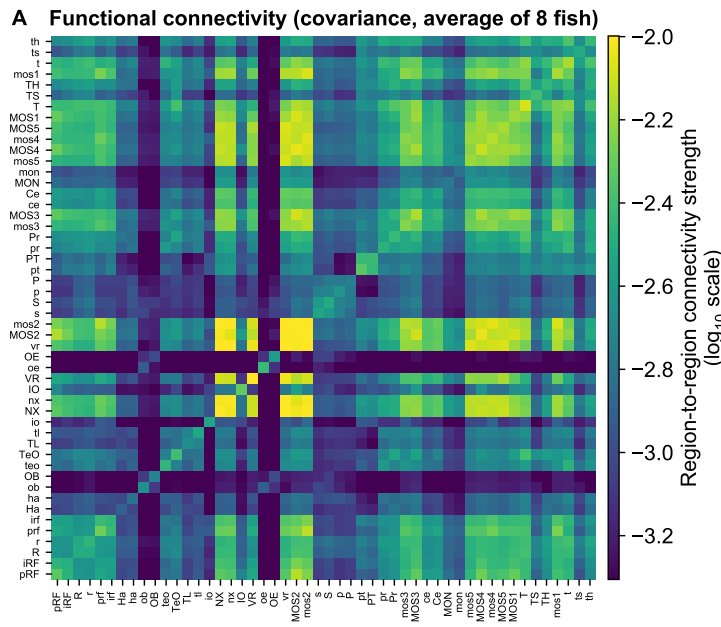

C

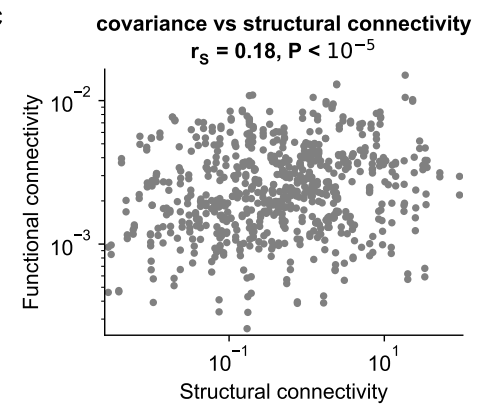

E

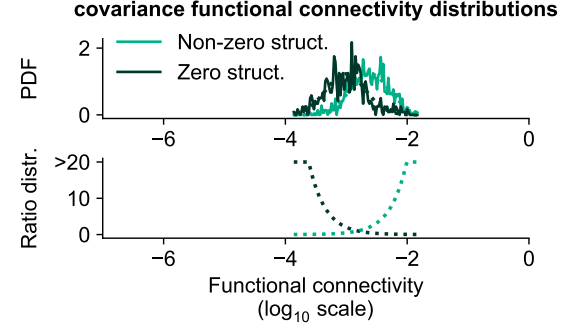

B Functional connectivity (correlation, average of 8 fish)

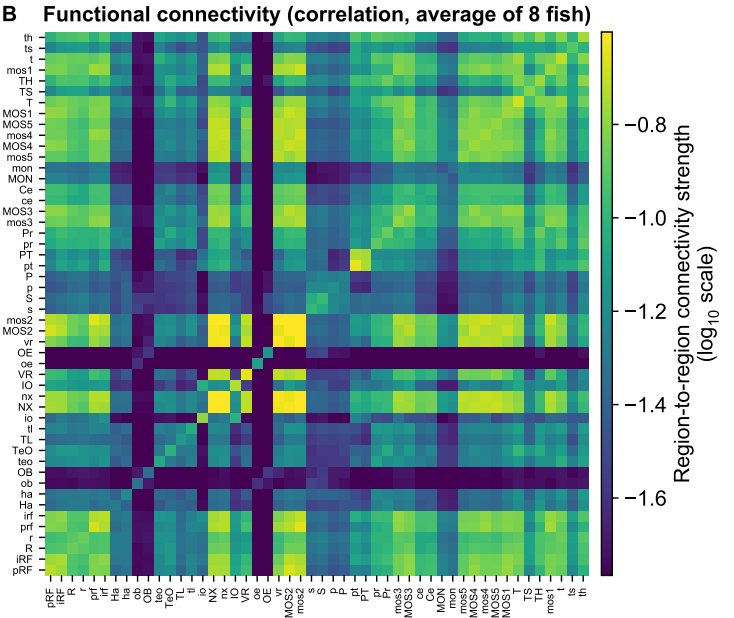

D correlation vs structural connectivity

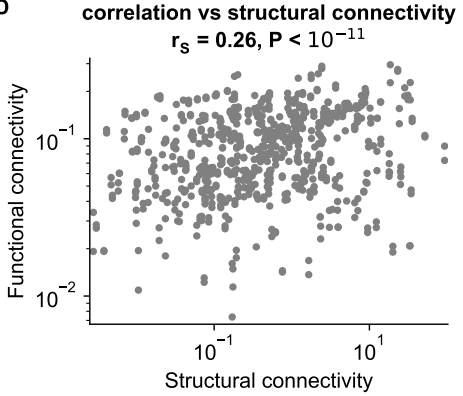

F correlation functional connectivity distributions

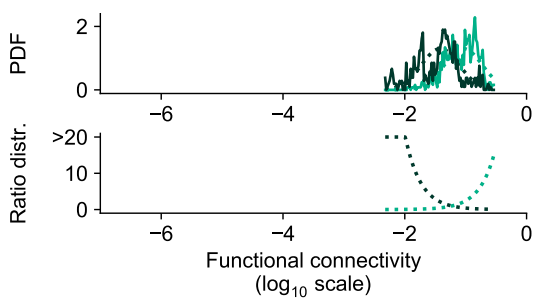

Figure S6: cRBM functional connectivity compared to baseline methods

(A) Average functional connectivity matrix of the covariance baseline method (see STAR methods) in $\log _{10}$ scale.

(B) Average functional connectivity matrix of the Pearson correlations baseline method (see STAR methods) in $\log _{10}$ scale.

(C, D) Equivalent figure to Figure 6C for functional connections as determined by the covariance (C) and correlations (D) between neurons (see STAR methods). The Spearman correlation coefficient between functional and structural connections for covariance is $r_{\mathrm{S}}=0.18$ and for correlation $r_{\mathrm{S}}=0.26$.

(E-F) Equivalent figure to Figure 6 for functional connections as determined by the covariance $(\mathrm{E})$ and correlations $(\mathrm{F})$ between neurons. Non-zero and zero distributions are significantly different (all $\mathrm{P}$ values $P<10^{-15}$, two-sided Kolmogorov-Smirnov tests). 


\section{References}

Abramowitz, M., Stegun, I. A., \& Romer, R. H. (1988). Handbook of mathematical functions with formulas, graphs, and mathematical tables.

Ahrens, M. B. (2019). Zebrafish neuroscience: Using artificial neural networks to help understand brains. Current Biology, 29, R1138-R1140.

Ahrens, M. B., \& Engert, F. (2015). Large-scale imaging in small brains. Current opinion in neurobiology, 32, 78-86.

Ahrens, M. B., Li, J. M., Orger, M. B., Robson, D. N., Schier, A. F., Engert, F., \& Portugues, R. (2012). Brain-wide neuronal dynamics during motor adaptation in zebrafish. Nature, 485, 471-477.

Ahrens, M. B., Orger, M. B., Robson, D. N., Li, J. M., \& Keller, P. J. (2013). Whole-brain functional imaging at cellular resolution using light-sheet microscopy. Nature methods, 10, 413-420.

Bargmann, C. I., \& Marder, E. (2013). From the connectome to brain function. Nature methods, 10, $483-490$.

Bassett, D. S., \& Sporns, O. (2017). Network neuroscience. Nature neuroscience, 20, 353-364.

Bathellier, B., Ushakova, L., \& Rumpel, S. (2012). Discrete neocortical dynamics predict behavioral categorization of sounds. Neuron, 76, 435-449.

Beretta, C. A., Dross, N., Gutierrez-Triana, J. A., Ryu, S., \& Carl, M. (2012). Habenula circuit development: past, present, and future. Frontiers in neuroscience, 6, 51.

Bialek, W. (2012). Biophysics: searching for principles. Princeton University Press.

Bishop, C. M. (2006). Pattern recognition and machine learning. springer.

Buzsáki, G. (2010). Neural syntax: cell assemblies, synapsembles, and readers. Neuron, 68, 362-385.

Chen, X., Mu, Y., Hu, Y., Kuan, A. T., Nikitchenko, M., Randlett, O., Chen, A. B., Gavornik, J. P., Sompolinsky, H., Engert, F. et al. (2018). Brain-wide organization of neuronal activity and convergent sensorimotor transformations in larval zebrafish. Neuron, 100, 876-890.

Chen, X., Randi, F., Leifer, A. M., \& Bialek, W. (2019). Searching for collective behavior in a small brain. Physical Review E, 99, 052418.

Das, A., \& Fiete, I. R. (2020). Systematic errors in connectivity inferred from activity in strongly recurrent networks. Nature Neuroscience, 23, 1286-1296.

Diana, G., Sainsbury, T. T., \& Meyer, M. P. (2019). Bayesian inference of neuronal assemblies. PLoS computational biology, 15, e1007481.

Dunn, T. W., Mu, Y., Narayan, S., Randlett, O., Naumann, E. A., Yang, C.-T., Schier, A. F., Freeman, J., Engert, F., \& Ahrens, M. B. (2016). Brain-wide mapping of neural activity controlling zebrafish exploratory locomotion. Elife, 5, e12741.

Eichenbaum, H. (2018). Barlow versus hebb: When is it time to abandon the notion of feature detectors and adopt the cell assembly as the unit of cognition? Neuroscience letters, 680, 88-93.

Ferrari, U., Obuchi, T., \& Mora, T. (2017). Random versus maximum entropy models of neural population activity. Physical Review E, 95, 042321.

Friedman, J., Hastie, T., \& Tibshirani, R. (2008). Sparse inverse covariance estimation with the graphical lasso. Biostatistics, 9, 432-441. 
Gardella, C., Marre, O., \& Mora, T. (2017). Restricted boltzmann machines provide an accurate metric for retinal responses to visual stimuli. In 5th International Conference on Learning Representations, ICLR 2017, Toulon, France, April 24-26, 2017, Workshop Track Proceedings. OpenReview.net. URL: https: //openreview .net/forum?id=Sk100nNFx

Gardella, C., Marre, O., \& Mora, T. (2019). Modeling the correlated activity of neural populations: A review. Neural computation, 31, 233-269.

Gerstein, G. L., Bedenbaugh, P., \& Aertsen, A. M. (1989). Neuronal assemblies. IEEE Transactions on Biomedical Engineering, 36, 4-14.

Harris, C. R., Millman, K. J., van der Walt, S. J., Gommers, R., Virtanen, P., Cournapeau, D., Wieser, E., Taylor, J., Berg, S., Smith, N. J., Kern, R., Picus, M., Hoyer, S., van Kerkwijk, M. H., Brett, M., Haldane, A., Fernández del Río, J., Wiebe, M., Peterson, P., Gérard-Marchant, P., Sheppard, K., Reddy, T., Weckesser, W., Abbasi, H., Gohlke, C., \& Oliphant, T. E. (2020). Array programming with NumPy. Nature, 585, 357-362. doi $10.1038 / \mathrm{s} 41586-020-2649-2$

Harris, K. D. (2005). Neural signatures of cell assembly organization. Nature Reviews Neuroscience, 6, 399407.

Harris, K. D. (2012). Cell assemblies of the superficial cortex. Neuron, 76, 263-265.

Hebb, D. O. (1949). The organization of behavior: A neuropsychological theory. Wiley.

Helmstaedter, M. (2015). The mutual inspirations of machine learning and neuroscience. Neuron, 86, 25-28.

Hinton, G. E. (2002). Training products of experts by minimizing contrastive divergence. Neural computation, $14,1771-1800$.

Hinton, G. E. (2012). A practical guide to training restricted boltzmann machines. In Neural networks: Tricks of the trade (pp. 599-619). Springer.

Hinton, G. E., \& Salakhutdinov, R. R. (2006). Reducing the dimensionality of data with neural networks. Science, 313, 504-507.

Hunter, J. D. (2007). Matplotlib: A 2d graphics environment. IEEE Annals of the History of Computing, 9, 90-95.

Jaynes, E. T. (1957). Information theory and statistical mechanics. Physical review, 106, 620.

Jazayeri, M., \& Afraz, A. (2017). Navigating the neural space in search of the neural code. Neuron, 93, $1003-1014$.

Kingma, D. P., \& Ba, J. (2014). Adam: A method for stochastic optimization. arXiv preprint arXiv:1412.6980,

Kopell, N. J., Gritton, H. J., Whittington, M. A., \& Kramer, M. A. (2014). Beyond the connectome: the dynome. Neuron, 83, 1319-1328.

Köster, U., Sohl-Dickstein, J., Gray, C. M., \& Olshausen, B. A. (2014). Modeling higher-order correlations within cortical microcolumns. PLoS computational biology, 10, e1003684.

Kunst, M., Laurell, E., Mokayes, N., Kramer, A., Kubo, F., Fernandes, A. M., Förster, D., Dal Maschio, M., \& 885 Baier, H. (2019). A cellular-resolution atlas of the larval zebrafish brain. Neuron, 103, 21-38.

Lam, S. K., Pitrou, A., \& Seibert, S. (2015). Numba: A llvm-based python jit compiler. In Proceedings of the Second Workshop on the LLVM Compiler Infrastructure in HPC (pp. 1-6). 
Lin, L., Osan, R., Shoham, S., Jin, W., Zuo, W., \& Tsien, J. Z. (2005). Identification of network-level coding units for real-time representation of episodic experiences in the hippocampus. Proceedings of the National Academy of Sciences, 102, 6125-6130.

Ma, L.-H., Grove, C. L., \& Baker, R. (2014). Development of oculomotor circuitry independent of hox3 genes. Nature communications, 5, 1-12.

Marques, J. C., Li, M., Schaak, D., Robson, D. N., \& Li, J. M. (2020). Internal state dynamics shape brainwide activity and foraging behaviour. Nature, 577, 239-243.

McKinney, W. et al. (2010). Data structures for statistical computing in python. In Proceedings of the 9th Python in Science Conference (pp. 51-56). Austin, TX volume 445.

Meshulam, L., Gauthier, J. L., Brody, C. D., Tank, D. W., \& Bialek, W. (2017). Collective behavior of place and non-place neurons in the hippocampal network. Neuron, 96, 1178-1191.

Migault, G., van der Plas, T. L., Trentesaux, H., Panier, T., Candelier, R., Proville, R., Englitz, B., Debrégeas, G., \& Bormuth, V. (2018). Whole-brain calcium imaging during physiological vestibular stimulation in larval zebrafish. Current Biology, 28, 3723-3735.

Mölter, J., Avitan, L., \& Goodhill, G. J. (2018). Detecting neural assemblies in calcium imaging data. BMC biology, 16, 1-20.

Mu, Y., Bennett, D. V., Rubinov, M., Narayan, S., Yang, C.-T., Tanimoto, M., Mensh, B. D., Looger, L. L., \& Ahrens, M. B. (2019). Glia accumulate evidence that actions are futile and suppress unsuccessful behavior. Cell, 178, 27-43.

Narayanan, N. S., Kimchi, E. Y., \& Laubach, M. (2005). Redundancy and synergy of neuronal ensembles in motor cortex. Journal of Neuroscience, 25, 4207-4216.

Palm, G., Knoblauch, A., Hauser, F., \& Schüz, A. (2014). Cell assemblies in the cerebral cortex. Biological cybernetics, 108, 559-572.

Panier, T., Romano, S., Olive, R., Pietri, T., Sumbre, G., Candelier, R., \& Debrégeas, G. (2013). Fast functional imaging of multiple brain regions in intact zebrafish larvae using selective plane illumination microscopy. Frontiers in neural circuits, 7, 65.

Pedregosa, F., Varoquaux, G., Gramfort, A., Michel, V., Thirion, B., Grisel, O., Blondel, M., Prettenhofer, P., Weiss, R., Dubourg, V. et al. (2011). Scikit-learn: Machine learning in python. the Journal of machine Learning research, 12, 2825-2830.

Posani, L., Cocco, S., \& Monasson, R. (2018). Integration and multiplexing of positional and contextual information by the hippocampal network. PLoS computational biology, 14, e1006320.

Quirin, S., Vladimirov, N., Yang, C.-T., Peterka, D. S., Yuste, R., \& Ahrens, M. B. (2016). Calcium imaging of neural circuits with extended depth-of-field light-sheet microscopy. Optics letters, 41, 855-858.

Randlett, O., Wee, C. L., Naumann, E. A., Nnaemeka, O., Schoppik, D., Fitzgerald, J. E., Portugues, R., Lacoste, A. M., Riegler, C., Engert, F. et al. (2015). Whole-brain activity mapping onto a zebrafish brain atlas. Nature methods, 12, 1039-1046.

Ravikumar, P., Wainwright, M. J., Lafferty, J. D. et al. (2010). High-dimensional ising model selection using 925 11-regularized logistic regression. The Annals of Statistics, 38, 1287-1319.

Romano, S. A., Pietri, T., Pérez-Schuster, V., Jouary, A., Haudrechy, M., \& Sumbre, G. (2015). Spontaneous neuronal network dynamics reveal circuit's functional adaptations for behavior. Neuron, 85, 1070-1085. 
Roussel, C., Cocco, S., \& Monasson, R. (2021). Barriers and dynamical paths in alternating gibbs sampling of restricted boltzmann machines. arXiv preprint arXiv:2107.06013, .

Vanwalleghem, G. C., Ahrens, M. B., \& Scott, E. K. (2018). Integrative whole-brain neuroscience in larval zebrafish. Current opinion in neurobiology, 50, 136-145.

Virtanen, P., Gommers, R., Oliphant, T. E., Haberland, M., Reddy, T., Cournapeau, D., Burovski, E., Peterson, P., Weckesser, W., Bright, J., van der Walt, S. J., Brett, M., Wilson, J., Millman, K. J., Mayorov, N., Nelson, D., Perktold, J., Cimrman, R., Henriksen, I., Quintero, E. A., Harris, C. R., Archibald, A. M., Ribeiro, A. H., Pedregosa, F., van Mulbregt, P., \& SciPy 1.0 Contributors (2020). SciPy 1.0: Fundamental Algorithms for Scientific Computing in Python. Nature Methods, 17, 261-272. doi:10.1038/s41592-019-0686-2 
Vladimirov, N., Mu, Y., Kawashima, T., Bennett, D. V., Yang, C.-T., Looger, L. L., Keller, P. J., Freeman, J., \& 970 Ahrens, M. B. (2014). Light-sheet functional imaging in fictively behaving zebrafish. Nature methods, 11, 883-884.

Volpi, R., Zanotto, M., Maccione, A., Di Marco, S., Berdondini, L., Sona, D., \& Murino, V. (2020). Modeling a population of retinal ganglion cells with restricted boltzmann machines. Scientific reports, 10, 1-10.

Waskom, M. L. (2021). Seaborn: statistical data visualization. Journal of Open Source Software, 6, 3021.

White, J. G., Southgate, E., Thomson, J. N., \& Brenner, S. (1986). The structure of the nervous system of the nematode caenorhabditis elegans. Philos Trans R Soc Lond B Biol Sci, 314, 1-340.

Wolf, S., Dubreuil, A. M., Bertoni, T., Böhm, U. L., Bormuth, V., Candelier, R., Karpenko, S., Hildebrand, D. G., Bianco, I. H., Monasson, R. et al. (2017). Sensorimotor computation underlying phototaxis in zebrafish. Nature communications, 8, 1-12.

Wolf, S., Supatto, W., Debrégeas, G., Mahou, P., Kruglik, S. G., Sintes, J.-M., Beaurepaire, E., \& Candelier, R. (2015). Whole-brain functional imaging with two-photon light-sheet microscopy. Nature methods, 12, $379-380$. 